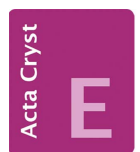

CRYSTALLOGRAPHIC COMMUNICATIONS

ISSN 2056-9890

Received 13 August 2018

Accepted 28 August 2018

Edited by M. Zeller, Purdue University, USA

Keywords: crystal structure; inorganic chemistry; organometallic chemistry; NHC; nickel; carbene.

CCDC reference: 1864396

Supporting information: this article has supporting information at journals.iucr.org/e

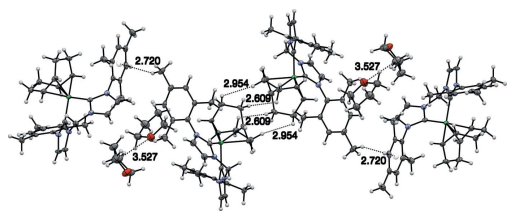

\section{Crystal structure of $\left(\eta^{4}\right.$-cyclooctadiene $)\left(3,3^{\prime}\right.$ -

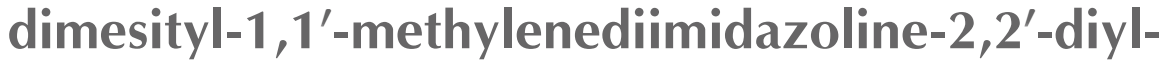 idene)nickel $(0)$ tetrahydrofuran monosolvate}

\author{
Carlos D. Yamamoto, Zijie Zhang and Sabine Chantal E. Stieber*
}

Department of Chemistry \& Biochemistry, California State Polytechnic University, Pomona, 3801 W. Temple Ave., Pomona, CA 91768, USA. *Correspondence e-mail: sestieber@cpp.edu

The crystal structure of the title compound, $\left[\mathrm{Ni}\left(\mathrm{C}_{25} \mathrm{H}_{28} \mathrm{~N}_{4}\right)\left(\mathrm{C}_{8} \mathrm{H}_{12}\right)\right] \cdot \mathrm{C}_{4} \mathrm{H}_{8} \mathrm{O}$ or $\left({ }^{\mathrm{Mes}} \mathrm{NHC}_{2} \mathrm{Me}\right) \mathrm{Ni}(\mathrm{COD})$, which contains a bidentate $\mathrm{N}$-heterocyclic carbene (NHC) ligand with mesityl aryl groups is reported. The complex at $100 \mathrm{~K}$ has monoclinic $\left(P 2_{1} / c\right)$ symmetry and a distorted tetrahedral geometry around the nickel center, with the cyclooctadiene ligand coordinated in a $\kappa^{2}, \eta^{2}$ fashion. The bidentate $\mathrm{NHC}$ ligand is not planar, with a $\mathrm{C}$ (carbene) $-\mathrm{Ni}-\mathrm{C}$ (carbene) angle of $91.51(12)^{\circ}$, resulting in the mesityl groups being on the same side of the cyclooctadiene (COD) ligand. One molecule of tetrahydrofuran (THF) is cocrystallized with the nickel complex and has positional disorder.

\section{Chemical context}

N-heterocyclic carbene (NHC) ligands, which have found extensive use in catalysis and organometallic chemistry, coordinate to metal centers via the lone pair of electrons of the carbene (Arduengo, 1999; Hopkinson et al., 2014; Lummiss et al., 2015). Bidentate $\mathrm{NHC}$ ligands $\left(\mathrm{NHC}_{2}\right)$ may be formed by linking two NHC ligands together; however, coordination to first row transition metals has been limited (Brendel et al., 2014; Herrmann et al., 1999; Douthwaite et al., 1999; Huffer et al., 2013; Harrold \& Hillhouse, 2013). Nickel(0)cyclooctadiene complexes with $\left\{1,1^{\prime}\right.$-di(isopropyl)phenyl-3, $3^{\prime}$-methylenediimidazolin-2,2'-diylidene $\}$ and $\left\{1,1^{\prime}\right.$-tert(butyl)-3,3'-methylenediimidazolin-2,2'-diylidene\} ligands have been reported, but the mesityl variant is not known (Brendel et al., 2014). Herein, a synthetic procedure for the synthesis of $\left\{1,1^{\prime}-\mathrm{di}\right.$ (mesityl)-3,3'-methylenediimidazolin-2,2' -diylidene\}nickel(0)cyclooctadiene, $\left({ }^{\mathrm{Mes}} \mathrm{NHC}_{2} \mathrm{Me}\right) \mathrm{Ni}(\mathrm{COD})$, and its crystallographic characterization are reported.

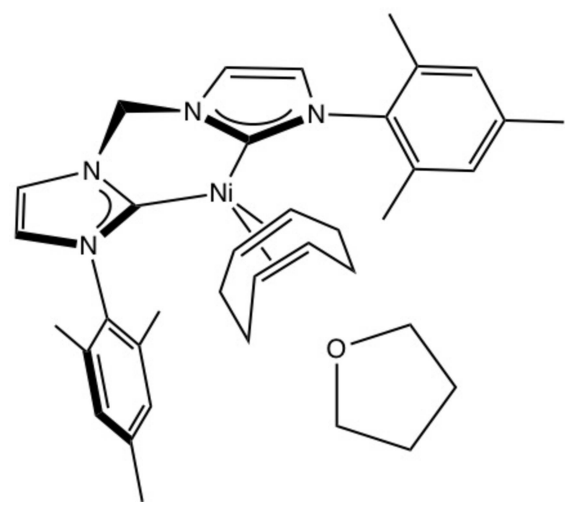

\section{Structural commentary}

( $\left.{ }^{\mathrm{Mes}} \mathrm{NHC}_{2} \mathrm{Me}\right) \mathrm{Ni}(\mathrm{COD})$ co-crystallizes with one molecule of tetrahydrofuran (THF) as shown in Fig. 1. Fig. 2 depicts the 
structure without the THF for clarity. The nickel(0) center has a pseudo-tetrahedral geometry, being coordinated to $\left({ }^{\text {Mes }} \mathrm{NHC}_{2} \mathrm{Me}\right.$ ) in a $\kappa^{2}$ fashion with a $\mathrm{C} 1-\mathrm{Ni} 1-\mathrm{C} 4$ angle of $91.51(12)^{\circ}$ and to COD in a $\kappa^{2}, \eta^{2}$ fashion. The distances between the nickel center and the $\left({ }^{\mathrm{Mes}} \mathrm{NHC}_{2} \mathrm{Me}\right)$ ligand are 1.909 (3) $\AA$ for Ni1-C1 and 1.916 (3) $\AA$ for Ni1-C4. These are slightly shorter than the analagous distances of 1.938 (3) and 1.953 (3) $\AA$, respectively, reported for ( $\left.{ }^{\text {Dipp }} \mathrm{NHC}_{2} \mathrm{Me}\right) \mathrm{Ni}(\mathrm{COD})$ (Brendel et al., 2014). The distances from the nickel center to the COD ligand are 1.921 (3) and 2.018 (3) $\AA$ as measured from Ni1 to the mid-points of $\mathrm{C} 29-$ $\mathrm{C} 30$ and $\mathrm{C} 26-\mathrm{C} 33$, respectively. The backbone of each $\mathrm{NHC}$ contains unsaturated $\mathrm{C}=\mathrm{C}$ double bonds, as evidenced by bond distances of 1.344 (4) $\AA$ for $\mathrm{C} 2-\mathrm{C} 3$ and 1.341 (4) $\AA$ for C5-C6. The other NHC backbone distances are 1.390 (4) $\AA$ for N1-C2, 1.384 (4) $\AA$ for N2-C3, 1.388 (3) $\AA$ for N3-C5, and 1.395 (3) $\AA$ for $\mathrm{N} 4-\mathrm{C} 6$. The remaining NHC bond lengths to the carbene are 1.377 (4) $\AA$ for $\mathrm{N} 1-\mathrm{C} 1,1.374$ (3) $\AA$ for N2-C1, 1.379 (3) $\AA$ for N3-C4, and 1.374 (3) $\AA$ for N4$\mathrm{C} 4$. These are comparable to the analagous NHC carbene distances reported for $\left({ }^{\text {Dipp }} \mathrm{NHC}_{2} \mathrm{Me}\right) \mathrm{Ni}(\mathrm{COD})$ of 1.374 (4), 1.387 (4), 1.379 (4), and 1.386 (4) $\AA$, respectively (Brendel et al., 2014). The portions of the COD ligand that are coordinated to nickel have $\mathrm{C}=\mathrm{C}$ bond distances of 1.411 (4) $\AA$ for $\mathrm{C} 29=\mathrm{C} 30$ and $1.374(4) \AA$ for $\mathrm{C} 26=\mathrm{C} 33$, consistent with unsaturated double bonds. These are slightly longer than the analagous $\mathrm{C}=\mathrm{C}$ COD distances reported for $\left({ }^{\text {Dipp }} \mathrm{NHC}_{2} \mathrm{Me}\right)$ $\mathrm{Ni}(\mathrm{COD})$ of 1.383 (5), and 1.355 (5) Å, respectively (Brendel et al., 2014). The remaining $\mathrm{C}-\mathrm{C}$ bond distances of the COD fragment are in the range of 1.512 (4)-1.539 (4) $\AA$, consistent with saturated $\mathrm{C}-\mathrm{C}$ single bonds, and comparable to the

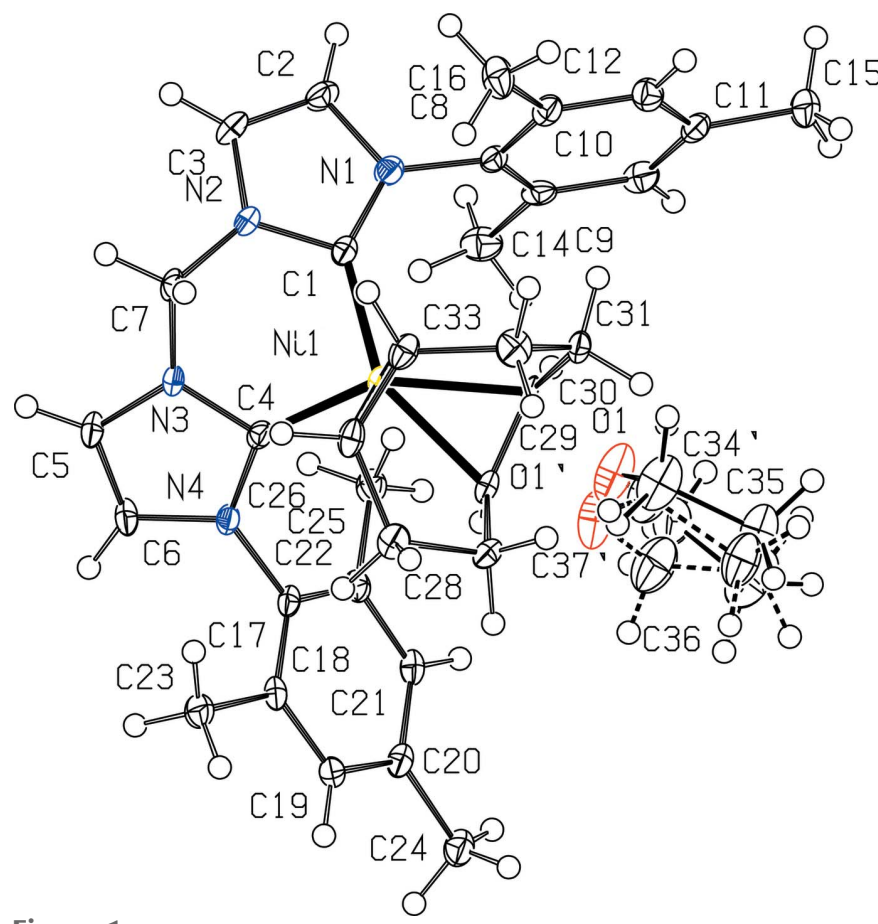

Figure 1

View of $\left({ }^{\mathrm{Mes}} \mathrm{NHC}_{2} \mathrm{Me}\right) \mathrm{Ni}(\mathrm{COD}) \cdot \mathrm{THF}$ with $50 \%$ probability ellipsoids, showing the THF disorder.

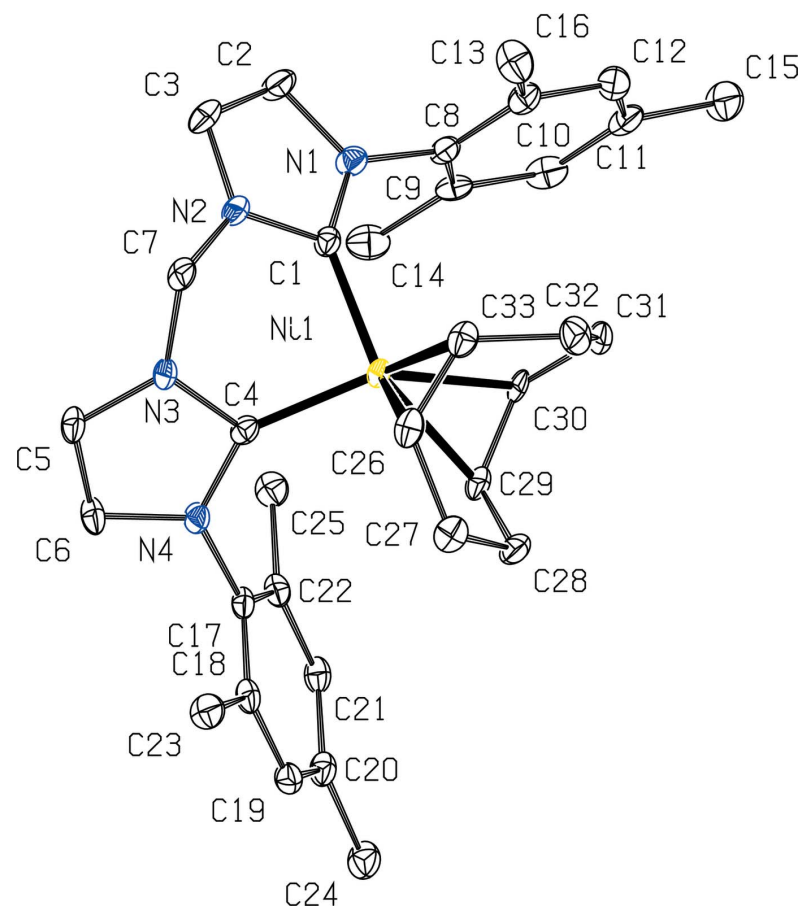

Figure 2

View of one molecule of $\left({ }^{\mathrm{Mes}} \mathrm{NHC}_{2} \mathrm{Me}\right) \mathrm{Ni}(\mathrm{COD})$ with $50 \%$ probability ellipsoids. The THF molecules and $\mathrm{H}$ atoms are omitted for clarity.

range of bond lengths reported for $\left({ }^{\text {Dipp }} \mathrm{NHC}_{2} \mathrm{Me}\right) \mathrm{Ni}(\mathrm{COD})$ of 1.502-1.529 А̊ (Brendel et al., 2014).

\section{Supramolecular features}

Four molecules of $\left({ }^{\mathrm{Mes}} \mathrm{NHC}_{2} \mathrm{Me}\right) \mathrm{Ni}(\mathrm{COD})$ and THF are present in the unit cell, as depicted in Fig. 3. The molecules are oriented such that the COD ligands from neighboring molecules are adjacent to each other, with distances of 2.61 and $2.95 \AA$ between nearest hydrogen atoms $(\mathrm{H} 28 A \cdots \mathrm{H} 32 A$ and $\mathrm{H} 27 B \cdots \mathrm{H} 31 B$, respectively). Standard deviations for distances including hydrogen atoms are not listed because hydrogen atoms were positionally fixed. The methyl group at the para position of the mesityl fragment is oriented towards the aryl ring of the mesityl of the neighboring molecule, with a distance of $2.72 \AA$ between the aryl ring centroid (C8-C13) and the nearest methyl hydrogen atom (H15C). The THF molecule is closest to the backbone of the $\left({ }^{\mathrm{Mes}} \mathrm{NHC}_{2} \mathrm{Me}\right)$ ligand, such that the molecules are 3.527 (17) $\AA$ apart from

Table 1

Intermolecular distances in the unit cell of $\left(\mathrm{MesNHC}_{2} \mathrm{Me}\right) \mathrm{Ni}(\mathrm{COD})$.

Standard deviations for distances including $\mathrm{H}$ atoms are omitted because $\mathrm{H}$ atoms were positionally fixed.

\begin{tabular}{|c|c|}
\hline & Distance $(\AA)$ \\
\hline H15C $\cdots$ centroid(C8-C13) & 2.72 \\
\hline $\mathrm{H} 27 B \cdots \mathrm{H} 31 B$ & 2.95 \\
\hline $\mathrm{H} 28 A \cdots \mathrm{H} 32 A$ & 2.61 \\
\hline $\mathrm{O} 1 \cdots \mathrm{C} 36$ & 3.527 (17) \\
\hline
\end{tabular}




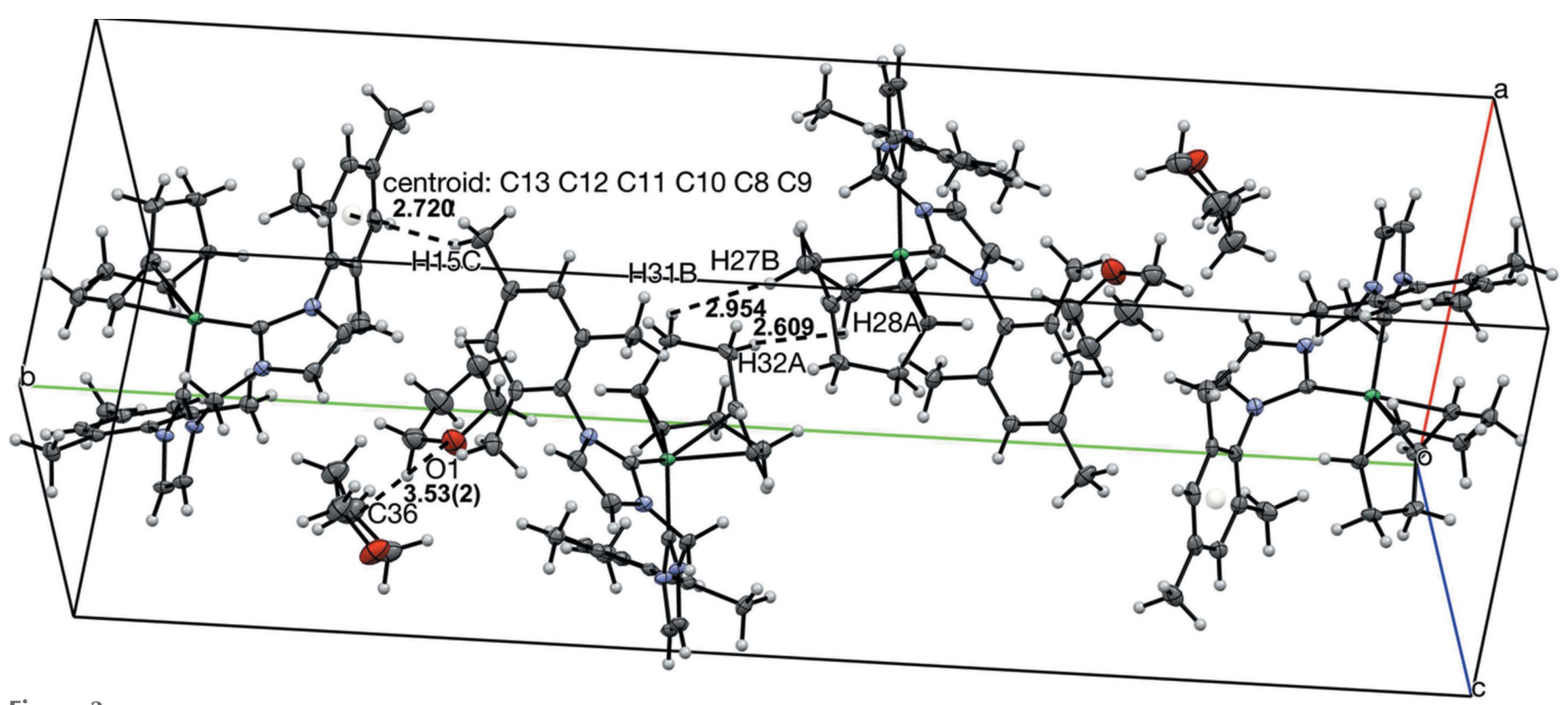

Figure 3

View of four molecules of $\left({ }^{\mathrm{Mes}} \mathrm{NHC}_{2} \mathrm{Me}\right) \mathrm{Ni}(\mathrm{COD})$ and $\mathrm{THF}$ in the unit cell with $50 \%$ probability ellipsoids, highlighting intermolecular distances. Distances between $\mathrm{H}$ atoms are listed without standard deviations because the $\mathrm{H}$ atoms were positionally fixed.

one oxygen atom $(\mathrm{O} 1)$ to the next nearest carbon atom (C36) (Table 1).

\section{Database survey}

A survey of the Cambridge Structural Database (Web accessed August 9, 2018; Groom et al., 2016) and SciFinder (SciFinder, 2018) yielded no exact matches for this complex, but related complexes with slightly varied ligands, such as $\left({ }^{\mathrm{tBu}} \mathrm{NHC}_{2} \mathrm{Me}\right) \mathrm{Ni}(\mathrm{COD})(\mathrm{tBu}=$ tert-butyl $)$ and $\left({ }^{\mathrm{Dipp}} \mathrm{NHC}_{2}\right.$ $\mathrm{Me}) \mathrm{Ni}(\mathrm{COD})(\mathrm{Dipp}=2,6-\mathrm{di}$ (isopropyl)phenyl) (Brendel et al., 2014) have been reported. The crystal structures of both these complexes have generally similar structural characteristics. The main difference is that the COD ligand in $\left({ }^{\mathrm{tBu}} \mathrm{NHC}_{2} \mathrm{Me}\right) \mathrm{Ni}(\mathrm{COD})$ is coordinated in a $\kappa^{1}, \eta^{2}$ fashion.

\section{Synthesis and crystallization}

1-(2,4,6-Trimethylphenyl)- $1 H$-imidazole, and 1,1'-di(mesityl)3,3'-methylene-diimidazolium dibromide were synthesized according to literature procedures (Liu et al., 2003; Gardiner et al., 1999). 1,1'-Di(mesityl)-3,3'-methylene-diimidazolium dibromide was dried overnight on a high vacuum line before transferring to an inert atmosphere $\mathrm{N}_{2}$ glovebox. $\left\{1,1^{\prime}-\mathrm{Di}\right.$ (mesityl)-3,3'-methylenediimidazolin-2,2'-diylidene\}nickel(0)cyclooctadiene was synthesized by the following method. A $20 \mathrm{~mL}$ scintillation vial was charged with $0.203 \mathrm{~g}(0.366 \mathrm{mmol}$, 1 eq.) of 1,1'-di(mesityl)-3,3'-methylene-diimidazolium dibromide, approximately $10 \mathrm{~mL}$ of tetrahydrofuran and a stirbar. $1.80 \mathrm{~mL}$ ( $0.915 \mathrm{mmol}, 2.5$ eq.) of $0.5 \mathrm{M}$ potassium bis(trimethylsilyl)amide in toluene were added dropwise to the solution while stirring, resulting in a color change to bluegreen. The mixture was stirred for approximately five h, resulting in a clear orange-brown solution, which was filtered through a glass frit with celite. The filtrate was transferred to a new $20 \mathrm{~mL}$ glass scintillation vial and stirred while adding $0.090 \mathrm{~g} \quad(0.329 \mathrm{mmol}, \quad 0.9$ eq. $)$ of bis(1,5-cyclooctadiene)nickel(0). The mixture was stirred for $4-12 \mathrm{~h}$, resulting in a clear dark red-orange solution. The solvent was removed in vacuo, and the orange solid was washed with pentane (3-5 washes of approximately $10 \mathrm{~mL})$, resulting in $0.151 \mathrm{~g}(78 \%)$ of an orange solid identified as $\left\{1,1^{\prime}\right.$-di(mesityl)-3, $3^{\prime}$-methylenediimidazolin-2,2'-diylidene\}nickel(0)cyclooctadiene.

Single crystals suitable for X-ray analysis were grown from a dilute solution of pentane with a drop of tetrahydrofuran. ${ }^{1} \mathrm{H}$ NMR (399.777 MHz, $\left.\mathrm{C}_{6} \mathrm{D}_{6}, 295 \mathrm{~K}\right): \delta=1.96-2.12\left(m, 8 \mathrm{H} ; \mathrm{CH}_{2}-\right.$ COD), 2.13 (s, 6H; $\mathrm{CH}_{3} p$-mesityl), $2.17\left(s, 12 \mathrm{H} ; \mathrm{CH}_{3} o\right.$ mesityl), 4.07 ( $s, 4 \mathrm{H}$; CH-Ni-COD), $4.68\left(s, 2 \mathrm{H}, \mathrm{CH}_{2}\right), 6.12(s$, 2H, CH-Im), 6.42 (s, 2H, CH-Im), $6.84(s, 4 \mathrm{H}, m-\mathrm{CH}-\mathrm{Ar}) .{ }^{13} \mathrm{C}$ NMR (101 MHz, $\left.\mathrm{C}_{6} \mathrm{D}_{6}, 295 \mathrm{~K}\right): \delta=18.43\left(\mathrm{CH}_{3} o\right.$-mesityl), $21.14\left(\mathrm{CH}_{3}\right.$ p-mesityl), $32.51\left(\mathrm{CH}_{2}\right.$-COD $), 61.31\left(\mathrm{CH}_{2}\right), 74.17$ (CH-Ni-COD), 118.18 (CH-Im), 119.81 (CH-Im), 128.94 (m$C \mathrm{H}-A r), 136.22$ (o-C-Ar), 137.89 (p-C-Ar), 138.91 (i-C-Ar), $205.37\left(\mathrm{~N}_{2} C-\mathrm{Im}\right)$.

\section{Refinement}

Crystal data, data collection and structure refinement details are summarized in Table 2. Most hydrogen atoms were placed in calculated positions using the AFIX commands of SHELXL and refined as riding with distances of $0.95 \AA$ for $\mathrm{C}-\mathrm{H}, 0.99 \AA$ for $\mathrm{CH}_{2}$ and $0.98 \AA$ for $\mathrm{CH}_{3}$. Methyl $\mathrm{H}$ atoms were allowed to rotate but not to tip to best fit the experimental electron density. $U_{\text {iso }}$ values of riding $\mathrm{H}$ atoms were set to 1.2 times $U_{\text {eq }}(\mathrm{C})$ for $\mathrm{CH}$ and $\mathrm{CH}_{2}$, and 1.5 times $U_{\text {eq }}(\mathrm{C})$ for $\mathrm{CH}_{3}$. The positions of the hydrogen atoms on the portions of the COD ligand directly bound to nickel and attached to C26, C29, C30, and C33 were determined from the difference map. 
Positions and isotropic displacement parameters were refined, but the associated $\mathrm{C}-\mathrm{H}$ atom distances were restrained to be similar to each other by using a SADI command of SHELXL (for $\mathrm{C} 26-\mathrm{H} 26 A, \mathrm{C} 29-\mathrm{H} 29 A, \mathrm{C} 30-\mathrm{H} 30 A$, and $\mathrm{C} 33-$ $\mathrm{H} 33 A)$.

The two moieties of the disordered THF molecule were restrained to have similar geometries (a SAME command in SHELXL was applied for $\mathrm{O}^{\prime}$ through $\mathrm{C} 37^{\prime}$ and $\mathrm{O} 1$ through C34 to make bond distances and angles equivalent with standard deviations of 0.02 and $0.04 \AA$ for 1,2 - and 1,3 distances, respectively). $U^{\mathrm{ij}}$ components of ADPs of the disordered atoms were restrained to be similar to each other with an esd of $0.01 \AA^{2}$ for atoms closer to each other than $2.0 \AA$ (SIMU command of SHELXL), resulting in a final close-to-equal site occupancy ratio of 0.502 (13) to 0.498 (13).

\section{Acknowledgements}

Special thanks to Charles Campana for helpful discussions.

\section{Funding information}

Funding for this research was provided by: U.S. Department of Defense, Army Research Office (grant No. W911NF-17-10537); California State University Program for Education Research in Biotechnology (CSUPERB); California State Polytechnic University, Pomona.

\section{References}

Arduengo, A. J. (1999). Acc. Chem. Res. 32, 913-921.

Brendel, M., Braun, C., Rominger, F. \& Hofmann, P. (2014). Angew. Chem. Int. Ed. 53, 8741-8745.

Bruker (2017). APEX3 and SAINT. Bruker AXS Inc., Madison, Wisconsin, USA.

Douthwaite, R. E., Haüssinger, D., Green, M. L. H., Silcock, P. J., Gomes, P. T., Martins, A. M. \& Danopoulos, A. A. (1999). Organometallics, 18, 4584-4590.

Gardiner, M. G., Herrmann, W. A., Reisinger, C., Schwarz, J. \& Spiegler, M. (1999). J. Organomet. Chem. 572, 239-247.

Groom, C. R., Bruno, I. J., Lightfoot, M. P. \& Ward, S. C. (2016). Acta Cryst. B72, 171-179.

Harrold, N. D. \& Hillhouse, G. L. (2013). Chem. Sci. 4, 4011-4015.

Herrmann, W. A., Schwarz, J., Gardiner, M. G. \& Spiegler, M. (1999). J. Organomet. Chem. 575, 80-86.

Hopkinson, M. N., Richter, C., Schedler, M. \& Glorius, F. (2014). Nature, 510, 485-496.

Huffer, A., Jeffery, B., Waller, B. J. \& Danopoulos, A. A. (2013). C. $R$. Chim. 16, 557-565.
Table 2

Experimental details.

\begin{tabular}{|c|c|}
\hline \multicolumn{2}{|l|}{ Crystal data } \\
\hline Chemical formula & {$\left[\mathrm{Ni}\left(\mathrm{C}_{25} \mathrm{H}_{28} \mathrm{~N}_{4}\right)\left(\mathrm{C}_{8} \mathrm{H}_{12}\right)\right] \cdot \mathrm{C}_{4} \mathrm{H}_{8} \mathrm{O}$} \\
\hline$M_{\mathrm{r}}$ & 623.50 \\
\hline Crystal system, space group & Monoclinic, $P 2_{1} / c$ \\
\hline Temperature $(\mathrm{K})$ & 100 \\
\hline$a, b, c(\AA)$ & $10.5557(7), 35.308(2), 8.5951(5)$ \\
\hline$\beta\left(^{\circ}\right)$ & $99.591(2)$ \\
\hline$V\left(\AA^{3}\right)$ & $3158.6(3)$ \\
\hline$Z$ & 4 \\
\hline Radiation type & Мо $K \alpha$ \\
\hline$\mu\left(\mathrm{mm}^{-1}\right)$ & 0.65 \\
\hline Crystal size $(\mathrm{mm})$ & $0.53 \times 0.15 \times 0.04$ \\
\hline \multicolumn{2}{|l|}{ Data collection } \\
\hline Diffractometer & Bruker D8 Venture Kappa \\
\hline Absorption correction & $\begin{array}{l}\text { Multi-scan (SADABS; Krause et } \\
\quad \text { al., 2015) }\end{array}$ \\
\hline$T_{\min }, T_{\max }$ & $0.658,0.746$ \\
\hline $\begin{array}{l}\text { No. of measured, independent and } \\
\text { observed }[I>2 \sigma(I)] \text { reflections }\end{array}$ & $61849,6973,4784$ \\
\hline$R_{\text {int }}$ & 0.141 \\
\hline$(\sin \theta / \lambda)_{\max }\left(\AA^{-1}\right)$ & 0.642 \\
\hline \multicolumn{2}{|l|}{ Refinement } \\
\hline$R\left[F^{2}>2 \sigma\left(F^{2}\right)\right], w R\left(F^{2}\right), S$ & $0.048,0.104,1.03$ \\
\hline No. of reflections & 6973 \\
\hline No. of parameters & 456 \\
\hline No. of restraints & 182 \\
\hline $\mathrm{H}$-atom treatment & $\begin{array}{l}\mathrm{H} \text { atoms treated by a mixture of } \\
\text { independent and constrained } \\
\text { refinement }\end{array}$ \\
\hline$\Delta \rho_{\max }, \Delta \rho_{\min }\left(\mathrm{e} \AA^{-3}\right)$ & $0.50,-0.46$ \\
\hline
\end{tabular}

Computer programs: APEX3 and SAINT (Bruker, 2017), SHELXT2014 (Sheldrick, 2015a), SHELXL2016 (Sheldrick, 2015b), Mercury (Macrae et al., 2006) and publCIF (Westrip, 2010).

Krause, L., Herbst-Irmer, R., Sheldrick, G. M. \& Stalke, D. (2015). J. Appl. Cryst. 48, 3-10.

Liu, J., Chen, J., Zhao, J., Zhao, Y., Li, L. \& Zhang, H. (2003). Synthesis, pp. 2661-2666.

Lummiss, J. A. M., Higman, C. S., Fyson, D. L., McDonald, R. \& Fogg, D. E. (2015). Chem. Sci. 6, 6739-6746.

Macrae, C. F., Edgington, P. R., McCabe, P., Pidcock, E., Shields, G. P., Taylor, R., Towler, M. \& van de Streek, J. (2006). J. Appl. Cryst. 39, 453-457.

SciFinder (2018). Chemical Abstracts Service: Colombus, OH, 2010; RN 58-08-2 (accessed August 10, 2018).

Sheldrick, G. M. (2015a). Acta Cryst. A71, 3-8.

Sheldrick, G. M. (2015b). Acta Cryst. C71, 3-8.

Westrip, S. P. (2010). J. Appl. Cryst. 43, 920-925. 


\section{supporting information}

Acta Cryst. (2018). E74, 1396-1399 [https://doi.org/10.1107/S2056989018012252]

Crystal structure of ( $\eta^{4}$-cyclooctadiene)(3,3'-dimesityl-1,1'-methylenediimidazoline-2,2'-diylidene)nickel(0) tetrahydrofuran monosolvate

\section{Carlos D. Yamamoto, Zijie Zhang and Sabine Chantal E. Stieber}

Computing details

Data collection: APEX3 (Bruker, 2017); cell refinement: SAINT (Bruker, 2017); data reduction: SAINT (Bruker, 2017); program(s) used to solve structure: SHELXT2014 (Sheldrick, 2015a); program(s) used to refine structure: SHELXL2016 (Sheldrick, 2015b); molecular graphics: Mercury (Macrae et al., 2006); software used to prepare material for publication: publCIF (Westrip, 2010).

( $\eta^{4}$-Cyclooctadiene)(3,3'-dimesityl-1,1'-methylenediimidazoline-2,2'-diylidene) nickel(0) tetrahydrofuran monosolvate

Crystal data

$\left[\mathrm{Ni}\left(\mathrm{C}_{25} \mathrm{H}_{28} \mathrm{~N}_{4}\right)\left(\mathrm{C}_{8} \mathrm{H}_{12}\right)\right] \cdot \mathrm{C}_{4} \mathrm{H}_{8} \mathrm{O}$

$F(000)=1336$

$M_{r}=623.50$

$D_{\mathrm{x}}=1.311 \mathrm{Mg} \mathrm{m}^{-3}$

Monoclinic, $P 2{ }_{1} / c$

Mo $K \alpha$ radiation, $\lambda=0.71073 \AA$

$a=10.5557$ (7) $\AA$

Cell parameters from 5806 reflections

$b=35.308(2) \AA$

$c=8.5951(5) \AA$

$\beta=99.591(2)^{\circ}$

$\theta=4.6-54.2^{\circ}$

$V=3158.6(3) \AA^{3}$

$\mu=0.65 \mathrm{~mm}^{-1}$

$T=100 \mathrm{~K}$

$Z=4$

Plate, orange

$0.53 \times 0.15 \times 0.04 \mathrm{~mm}$

\section{Data collection}

Bruker D8 Venture Kappa

6973 independent reflections

diffractometer

Radiation source: microfocus sealed tube 4784 reflections with $I>2 \sigma(I)$

$\varphi$ and $\omega$ scans

Absorption correction: multi-scan

(SADABS; Krause et al., 2015)

$T_{\min }=0.658, T_{\max }=0.746$

$R_{\text {int }}=0.141$

$\theta_{\max }=27.2^{\circ}, \theta_{\min }=2.6^{\circ}$

$h=-13 \rightarrow 13$

$k=-45 \rightarrow 45$

$l=-10 \rightarrow 11$

61849 measured reflections

Refinement

Refinement on $F^{2}$

Least-squares matrix: full

$R\left[F^{2}>2 \sigma\left(F^{2}\right)\right]=0.048$

$w R\left(F^{2}\right)=0.104$

$S=1.03$

6973 reflections

456 parameters

182 restraints

Primary atom site location: structure-invariant direct methods

Secondary atom site location: difference Fourier map

Hydrogen site location: mixed

$\mathrm{H}$ atoms treated by a mixture of independent and constrained refinement 
$w=1 /\left[\sigma^{2}\left(F_{\mathrm{o}}^{2}\right)+(0.0168 P)^{2}+5.0058 P\right]$

where $P=\left(F_{\mathrm{o}}^{2}+2 F_{\mathrm{c}}^{2}\right) / 3$

$(\Delta / \sigma)_{\max }<0.001$

$$
\Delta \rho_{\max }=0.50 \mathrm{e} \AA^{-3}
$$

$\Delta \rho_{\min }=-0.46$ e $\AA^{-3}$

\section{Special details}

Geometry. All esds (except the esd in the dihedral angle between two 1.s. planes) are estimated using the full covariance matrix. The cell esds are taken into account individually in the estimation of esds in distances, angles and torsion angles; correlations between esds in cell parameters are only used when they are defined by crystal symmetry. An approximate (isotropic) treatment of cell esds is used for estimating esds involving 1.s. planes.

Fractional atomic coordinates and isotropic or equivalent isotropic displacement parameters $\left(\AA^{2}\right)$

\begin{tabular}{|c|c|c|c|c|c|}
\hline & $x$ & $y$ & $z$ & $U_{\text {iso }} * / U_{\text {eq }}$ & Occ. $(<1)$ \\
\hline $\mathrm{Ni01}$ & $0.40659(3)$ & $0.59095(2)$ & $0.82589(4)$ & $0.01352(10)$ & \\
\hline $\mathrm{N} 1$ & $0.5497(2)$ & $0.66097(7)$ & 0.9799 & $0.0171(5)$ & \\
\hline $\mathrm{N} 2$ & $0.4717(2)$ & $0.62336(7)$ & $1.1329(3)$ & $0.0160(5)$ & \\
\hline N3 & $0.2805(2)$ & $0.58667(7)$ & $1.0943(3)$ & 0.0158 & \\
\hline N4 & $0.1305(2)$ & $0.58132(7)$ & 0.8953 & 0.0155 & \\
\hline $\mathrm{C} 1$ & $0.4848(3)$ & $0.62709(8)$ & $0.9772(3)$ & $0.0147(6)$ & \\
\hline $\mathrm{C} 2$ & $0.5758(3)$ & $0.67656(9)$ & 1.1304 (3) & $0.0217(7)$ & \\
\hline $\mathrm{H} 2$ & 0.620362 & 0.699526 & 1.159441 & $0.026^{*}$ & \\
\hline $\mathrm{C} 3$ & 0.5259 & $0.65294(9)$ & $1.2269(3)$ & $0.0209(7)$ & \\
\hline H3 & 0.527500 & 0.655931 & 1.337005 & $0.025^{*}$ & \\
\hline $\mathrm{C} 4$ & $0.2613(3)$ & $0.58607(8)$ & $0.9316(3)$ & $0.0144(6)$ & \\
\hline $\mathrm{C} 5$ & 0.1676 & $0.58212(8)$ & 1.1549 & $0.0179(6)$ & \\
\hline H5 & 0.158714 & 0.581583 & 1.263073 & $0.021^{*}$ & \\
\hline C6 & $0.0735(3)$ & $0.57865(8)$ & $1.0301(3)$ & $0.0184(6)$ & \\
\hline H6 & -0.015291 & 0.575044 & 1.032910 & $0.022 *$ & \\
\hline $\mathrm{C} 7$ & $0.4080(3)$ & $0.59040(9)$ & $1.1849(3)$ & $0.0165(6)$ & \\
\hline H7A & 0.458691 & 0.567380 & 1.171654 & $0.020^{*}$ & \\
\hline H7B & 0.402361 & 0.592985 & 1.298263 & $0.020 *$ & \\
\hline $\mathrm{C} 8$ & 0.5968 & $0.67746(8)$ & 0.8478 & $0.0163(6)$ & \\
\hline C9 & 0.5204 & $0.70228(8)$ & 0.7464 & $0.0177(6)$ & \\
\hline $\mathrm{C} 10$ & 0.5734 & $0.71794(8)$ & $0.6224(4)$ & $0.0214(7)$ & \\
\hline $\mathrm{H} 10$ & 0.521938 & 0.734348 & 0.550278 & $0.026^{*}$ & \\
\hline $\mathrm{C} 11$ & $0.6975(3)$ & $0.71046(8)$ & 0.6009 (3) & $0.0201(7)$ & \\
\hline $\mathrm{C} 12$ & 0.7709 (3) & $0.68572(8)$ & $0.7052(3)$ & $0.0200(7)$ & \\
\hline H12 & 0.856658 & 0.680319 & 0.691993 & $0.024 *$ & \\
\hline $\mathrm{C} 13$ & 0.7218 & $0.66878(8)$ & $0.8281(3)$ & 0.0178 & \\
\hline $\mathrm{C} 14$ & 0.3861 & $0.71230(9)$ & 0.7685 & $0.0262(7)$ & \\
\hline H14A & 0.330746 & 0.714082 & 0.665288 & $0.039 *$ & \\
\hline H14B & 0.387035 & 0.736703 & 0.823046 & $0.039 *$ & \\
\hline $\mathrm{H} 14 \mathrm{C}$ & 0.353120 & 0.692657 & 0.831690 & $0.039 *$ & \\
\hline $\mathrm{C} 15$ & 0.7554 & $0.72843(10)$ & $0.4696(4)$ & $0.0282(8)$ & \\
\hline H15A & 0.795053 & 0.708790 & 0.413047 & $0.042 *$ & \\
\hline H15B & 0.820795 & 0.746881 & 0.514294 & $0.042 *$ & \\
\hline $\mathrm{H} 15 \mathrm{C}$ & 0.687898 & 0.741271 & 0.396340 & $0.042 *$ & \\
\hline $\mathrm{C} 16$ & 0.8014 & $0.64084(9)$ & 0.9349 & $0.0253(7)$ & \\
\hline H16A & 0.753695 & 0.617055 & 0.936258 & $0.038 *$ & \\
\hline
\end{tabular}




\begin{tabular}{|c|c|c|c|c|c|}
\hline H16B & 0.820161 & 0.651211 & 1.042069 & $0.038^{*}$ & \\
\hline $\mathrm{H} 16 \mathrm{C}$ & 0.882059 & 0.636053 & 0.896079 & $0.038^{*}$ & \\
\hline $\mathrm{C} 17$ & $0.0553(3)$ & $0.58069(8)$ & 0.7392 & $0.0144(6)$ & \\
\hline C18 & -0.0086 & $0.54770(8)$ & $0.6843(3)$ & $0.0158(6)$ & \\
\hline C19 & -0.0857 (3) & $0.54843(8)$ & $0.5365(3)$ & $0.0169(6)$ & \\
\hline H19 & -0.129619 & 0.526032 & 0.497217 & $0.020^{*}$ & \\
\hline $\mathrm{C} 20$ & -0.1002 & $0.58104(8)$ & $0.4447(3)$ & $0.0171(6)$ & \\
\hline $\mathrm{C} 21$ & -0.0343 & $0.61332(8)$ & $0.5027(3)$ & $0.0171(6)$ & \\
\hline $\mathrm{H} 21$ & -0.042410 & 0.635603 & 0.439830 & $0.021 *$ & \\
\hline $\mathrm{C} 22$ & 0.0438 & $0.61413(8)$ & $0.6504(3)$ & $0.0167(6)$ & \\
\hline $\mathrm{C} 23$ & $-0.0007(3)$ & $0.51193(8)$ & $0.7823(3)$ & $0.0210(7)$ & \\
\hline $\mathrm{H} 23 \mathrm{~A}$ & -0.016531 & 0.489902 & 0.712349 & $0.032 *$ & \\
\hline H23B & -0.065404 & 0.512797 & 0.851747 & $0.032^{*}$ & \\
\hline $\mathrm{H} 23 \mathrm{C}$ & 0.085074 & 0.509905 & 0.846178 & $0.032 *$ & \\
\hline $\mathrm{C} 24$ & $-0.1874(3)$ & $0.58143(9)$ & $0.2862(3)$ & $0.0229(7)$ & \\
\hline $\mathrm{H} 24 \mathrm{~A}$ & -0.147939 & 0.566754 & 0.210144 & $0.034 *$ & \\
\hline H24B & -0.200454 & 0.607603 & 0.249046 & $0.034^{*}$ & \\
\hline $\mathrm{H} 24 \mathrm{C}$ & -0.270478 & 0.570174 & 0.296654 & $0.034^{*}$ & \\
\hline $\mathrm{C} 25$ & 0.1133 & $0.64962(8)$ & $0.7106(3)$ & $0.0201(7)$ & \\
\hline $\mathrm{H} 25 \mathrm{~A}$ & 0.206066 & 0.644930 & 0.729324 & $0.030^{*}$ & \\
\hline $\mathrm{H} 25 \mathrm{~B}$ & 0.086099 & 0.657291 & 0.809584 & $0.030^{*}$ & \\
\hline $\mathrm{H} 25 \mathrm{C}$ & 0.093035 & 0.669840 & 0.632288 & $0.030^{*}$ & \\
\hline $\mathrm{C} 26$ & $0.4432(3)$ & $0.53181(8)$ & $0.8337(3)$ & $0.0186(7)$ & \\
\hline H26A & $0.423(2)$ & $0.5236(7)$ & $0.932(2)$ & $0.010(7)^{*}$ & \\
\hline $\mathrm{C} 27$ & 0.3628 & $0.51489(9)$ & $0.6886(3)$ & $0.0208(7)$ & \\
\hline H27A & 0.276896 & 0.508545 & 0.713097 & $0.025^{*}$ & \\
\hline H27B & 0.403403 & 0.491004 & 0.661976 & $0.025^{*}$ & \\
\hline $\mathrm{C} 28$ & $0.3460(3)$ & $0.54110(8)$ & $0.5439(3)$ & $0.0192(7)$ & \\
\hline $\mathrm{H} 28 \mathrm{~A}$ & 0.417278 & 0.536693 & 0.484457 & $0.023^{*}$ & \\
\hline H28B & 0.264580 & 0.534738 & 0.473722 & $0.023^{*}$ & \\
\hline $\mathrm{C} 29$ & $0.3440(3)$ & $0.58277(8)$ & 0.5894 (3) & $0.0163(6)$ & \\
\hline H29A & $0.262(2)$ & $0.5947(8)$ & 0.559 & $0.014(8)^{*}$ & \\
\hline $\mathrm{C} 30$ & $0.4559(3)$ & $0.60531(8)$ & $0.6134(3)$ & $0.0149(6)$ & \\
\hline $\mathrm{H} 30 \mathrm{~A}$ & $0.443(3)$ & $0.6319(6)$ & $0.597(4)$ & $0.024(9)^{*}$ & \\
\hline $\mathrm{C} 31$ & $0.5874(3)$ & $0.59020(9)$ & $0.5982(3)$ & $0.0189(6)$ & \\
\hline H31A & 0.651953 & 0.610249 & 0.630734 & $0.023^{*}$ & \\
\hline H31B & 0.588887 & 0.584307 & 0.485938 & $0.023^{*}$ & \\
\hline $\mathrm{C} 32$ & $0.6263(3)$ & $0.55448(9)$ & 0.6975 (4) & $0.0230(7)$ & \\
\hline $\mathrm{H} 32 \mathrm{~A}$ & 0.607565 & 0.531937 & 0.629023 & $0.028^{*}$ & \\
\hline H32B & 0.720140 & 0.555157 & 0.735062 & $0.028^{*}$ & \\
\hline C33 & $0.5586(3)$ & $0.55016(9)$ & $0.8387(3)$ & $0.0192(7)$ & \\
\hline H33A & $0.612(2)$ & $0.5536(8)$ & $0.940(3)$ & $0.014(8)^{*}$ & \\
\hline $\mathrm{O} 1$ & $0.1468(10)$ & $0.7119(3)$ & $0.3805(9)$ & $0.0435(18)$ & $0.502(13)$ \\
\hline C34 & $0.2266(17)$ & $0.6845(6)$ & $0.3253(16)$ & $0.041(2)$ & $0.502(13)$ \\
\hline $\mathrm{H} 34 \mathrm{~A}$ & 0.200127 & 0.658717 & 0.351848 & $0.049^{*}$ & $0.502(13)$ \\
\hline H34B & 0.317128 & 0.688406 & 0.375222 & $0.049^{*}$ & $0.502(13)$ \\
\hline $\mathrm{C} 35$ & $0.2128(11)$ & 0.6890 (4) & $0.1491(15)$ & $0.037(2)$ & $0.502(13)$ \\
\hline $\mathrm{H} 35 \mathrm{~A}$ & 0.283351 & 0.704611 & 0.120317 & $0.045^{*}$ & $0.502(13)$ \\
\hline
\end{tabular}




\begin{tabular}{|c|c|c|c|c|c|}
\hline H35B & 0.212307 & 0.664092 & 0.096250 & $0.045^{*}$ & $0.502(13)$ \\
\hline C36 & $0.0855(17)$ & $0.7087(4)$ & 0.1057 (14) & $0.044(2)$ & $0.502(13)$ \\
\hline H36A & 0.014923 & 0.690064 & 0.079960 & $0.052 *$ & $0.502(13)$ \\
\hline H36B & 0.085608 & 0.725673 & 0.014167 & $0.052 *$ & $0.502(13)$ \\
\hline $\mathrm{C} 37$ & $0.0717(10)$ & $0.7310(3)$ & $0.2521(10)$ & 0.0352 (19) & $0.502(13)$ \\
\hline H37A & 0.103029 & 0.757211 & 0.244043 & $0.042 *$ & $0.502(13)$ \\
\hline H37B & -0.019410 & 0.731908 & 0.266236 & $0.042 *$ & $0.502(13)$ \\
\hline $\mathrm{O} 1^{\prime}$ & $0.0905(10)$ & $0.7022(2)$ & $0.3664(9)$ & $0.0391(17)$ & $0.498(13)$ \\
\hline C34' & $0.2015(16)$ & $0.6844(6)$ & $0.3267(16)$ & $0.041(2)$ & $0.498(13)$ \\
\hline $\mathrm{H} 34 \mathrm{C}$ & 0.213464 & 0.658950 & 0.375138 & $0.049 *$ & $0.498(13)$ \\
\hline H34D & 0.279163 & 0.699780 & 0.363667 & $0.049 *$ & $0.498(13)$ \\
\hline $\mathrm{C} 35^{\prime}$ & $0.1769(12)$ & $0.6816(3)$ & $0.1473(15)$ & $0.037(2)$ & $0.498(13)$ \\
\hline $\mathrm{H} 35 \mathrm{C}$ & 0.131992 & 0.657687 & 0.111634 & $0.045^{*}$ & $0.498(13)$ \\
\hline H35D & 0.258105 & 0.682994 & 0.104300 & $0.045 *$ & $0.498(13)$ \\
\hline C $36^{\prime}$ & $0.0919(16)$ & $0.7159(4)$ & 0.0979 (13) & $0.037(2)$ & $0.498(13)$ \\
\hline $\mathrm{H} 36 \mathrm{C}$ & 0.143422 & 0.738695 & 0.084198 & $0.044 *$ & $0.498(13)$ \\
\hline H36D & 0.030569 & 0.711026 & -0.000385 & $0.044^{*}$ & $0.498(13)$ \\
\hline C37' & $0.0240(12)$ & 0.7195 & $0.2392(11)$ & $0.046(2)$ & $0.498(13)$ \\
\hline $\mathrm{H} 37 \mathrm{C}$ & 0.013576 & 0.746664 & 0.263105 & $0.056^{*}$ & $0.498(13)$ \\
\hline H37D & -0.062699 & 0.708117 & 0.213787 & $0.056^{*}$ & $0.498(13)$ \\
\hline
\end{tabular}

Atomic displacement parameters $\left(\AA^{2}\right)$

\begin{tabular}{|c|c|c|c|c|c|c|}
\hline & $U^{11}$ & $U^{22}$ & $U^{33}$ & $U^{12}$ & $U^{13}$ & $U^{23}$ \\
\hline $\mathrm{Ni01}$ & $0.01553(18)$ & 0.01667 (19) & $0.00880(17)$ & $-0.00028(16)$ & $0.00334(13)$ & $-0.00042(16)$ \\
\hline N1 & $0.0191(13)$ & $0.0178(14)$ & $0.0137(12)$ & $-0.0008(10)$ & $0.0009(10)$ & $0.0001(10)$ \\
\hline $\mathrm{N} 2$ & $0.0189(13)$ & $0.0194(14)$ & $0.0095(12)$ & $-0.0007(10)$ & $0.0016(10)$ & $-0.0006(10)$ \\
\hline N3 & $0.0177(12)$ & $0.0209(14)$ & $0.0098(11)$ & $-0.0016(11)$ & $0.0050(9)$ & $0.0011(10)$ \\
\hline N4 & $0.0163(12)$ & $0.0202(14)$ & $0.0104(12)$ & $-0.0001(10)$ & $0.0033(10)$ & $0.0025(10)$ \\
\hline $\mathrm{C} 1$ & $0.0147(14)$ & $0.0183(16)$ & $0.0111(14)$ & $0.0021(12)$ & $0.0020(11)$ & $0.0004(12)$ \\
\hline $\mathrm{C} 2$ & $0.0287(18)$ & $0.0203(17)$ & $0.0153(15)$ & $-0.0043(14)$ & $0.0017(13)$ & $-0.0059(12)$ \\
\hline $\mathrm{C} 3$ & $0.0269(17)$ & $0.0241(17)$ & $0.0106(15)$ & $0.0016(14)$ & $-0.0001(13)$ & $-0.0035(12)$ \\
\hline $\mathrm{C} 4$ & $0.0180(14)$ & $0.0144(15)$ & $0.0105(13)$ & $-0.0003(12)$ & $0.0013(11)$ & $0.0012(11)$ \\
\hline $\mathrm{C} 5$ & $0.0197(15)$ & $0.0237(17)$ & $0.0116(14)$ & $0.0033(13)$ & $0.0067(12)$ & $0.0028(12)$ \\
\hline C6 & $0.0171(15)$ & $0.0243(17)$ & $0.0157(15)$ & $0.0035(12)$ & $0.0083(12)$ & 0.0057 (12) \\
\hline $\mathrm{C} 7$ & $0.0218(15)$ & $0.0195(15)$ & $0.0082(13)$ & $0.0002(13)$ & $0.0022(11)$ & $0.0042(12)$ \\
\hline $\mathrm{C} 8$ & $0.0214(15)$ & $0.0154(15)$ & $0.0118(14)$ & $-0.0042(12)$ & $0.0020(12)$ & $-0.0018(11)$ \\
\hline C9 & $0.0206(16)$ & $0.0125(15)$ & $0.0184(16)$ & $-0.0035(12)$ & $-0.0018(12)$ & $-0.0029(12)$ \\
\hline $\mathrm{C} 10$ & $0.0284(18)$ & $0.0139(16)$ & $0.0189(16)$ & $-0.0011(13)$ & $-0.0046(13)$ & $0.0050(12)$ \\
\hline $\mathrm{C} 11$ & $0.0309(18)$ & $0.0171(16)$ & $0.0114(15)$ & $-0.0062(13)$ & $0.0010(13)$ & $-0.0008(12)$ \\
\hline $\mathrm{C} 12$ & $0.0214(16)$ & 0.0193 (17) & $0.0196(16)$ & $-0.0003(13)$ & 0.0048 (13) & $-0.0003(13)$ \\
\hline $\mathrm{C} 13$ & $0.0232(16)$ & $0.0157(16)$ & $0.0139(15)$ & $0.0001(13)$ & $0.0016(12)$ & $0.0004(12)$ \\
\hline $\mathrm{C} 14$ & $0.0250(18)$ & $0.0223(18)$ & $0.0292(18)$ & $0.0006(14)$ & $-0.0018(14)$ & $0.0002(14)$ \\
\hline C15 & $0.037(2)$ & 0.0299 (19) & $0.0182(17)$ & $-0.0086(16)$ & $0.0055(14)$ & 0.0058 (14) \\
\hline $\mathrm{C} 16$ & $0.0259(17)$ & 0.0259 (19) & $0.0248(18)$ & $0.0037(14)$ & $0.0060(14)$ & $0.0118(14)$ \\
\hline $\mathrm{C} 17$ & 0.0130 & $0.0207(16)$ & $0.0104(14)$ & $0.0035(12)$ & $0.0041(11)$ & $0.0023(11)$ \\
\hline $\mathrm{C} 18$ & $0.0158(14)$ & $0.0192(16)$ & $0.0136(15)$ & $0.0020(12)$ & $0.0062(12)$ & $0.0045(12)$ \\
\hline C19 & $0.0165(15)$ & 0.0197 (16) & $0.0151(15)$ & $-0.0016(12)$ & $0.0047(12)$ & $0.0017(12)$ \\
\hline
\end{tabular}




$\begin{array}{lllllll}\text { C20 } & 0.0159(14) & 0.0233(17) & 0.0124(14) & 0.0048(12) & 0.0032(11) & 0.0022(12) \\ \text { C21 } & 0.0193(15) & 0.0189(16) & 0.0144(15) & 0.0030(12) & 0.0063(12) & 0.0048(12) \\ \text { C22 } & 0.0143(14) & 0.0203(16) & 0.0168(15) & 0.0014(12) & 0.0060(12) & 0.0011(12) \\ \text { C23 } & 0.0264(17) & 0.0196(17) & 0.0171(16) & -0.0029(13) & 0.0037(13) & 0.0041(13) \\ \text { C24 } & 0.0235(16) & 0.0286(19) & 0.0169(16) & 0.0020(14) & 0.0037(13) & 0.0030(13) \\ \text { C25 } & 0.0208(16) & 0.0214(16) & 0.0179(16) & 0.0013(13) & 0.0029(13) & 0.0041(12) \\ \text { C26 } & 0.0257(17) & 0.0169(16) & 0.0140(15) & 0.0060(13) & 0.0055(13) & 0.0046(12) \\ \text { C27 } & 0.0261(17) & 0.0171(16) & 0.0192(16) & 0.0018(13) & 0.0040(13) & 0.0006(13) \\ \text { C28 } & 0.0233(16) & 0.0219(17) & 0.0119(15) & -0.0025(13) & 0.0016(12) & -0.0030(12) \\ \text { C29 } & 0.0204(15) & 0.0205(17) & 0.0084(14) & 0.0026(13) & 0.0032(11) & 0.0009(11) \\ \text { C30 } & 0.0224(16) & 0.0180(16) & 0.0053(13) & 0.0006(12) & 0.0049(11) & 0.0006(11) \\ \text { C31 } & 0.0182(14) & 0.0286(17) & 0.0109(14) & -0.0019(14) & 0.0053(11) & 0.0001(13) \\ \text { C32 } & 0.0171(16) & 0.0274(18) & 0.0256(18) & 0.0070(13) & 0.0065(13) & -0.0021(14) \\ \text { C33 } & 0.0201(16) & 0.0231(17) & 0.0137(15) & 0.0072(13) & 0.0011(12) & 0.0013(13) \\ \text { O1 } & 0.061(4) & 0.047(4) & 0.024(3) & 0.020(3) & 0.011(3) & 0.005(3) \\ \text { C34 } & 0.054(5) & 0.037(4) & 0.031(3) & 0.019(4) & 0.007(3) & 0.002(3) \\ \text { C35 } & 0.047(4) & 0.040(4) & 0.026(3) & 0.013(4) & 0.010(3) & 0.004(3) \\ \text { C36 } & 0.048(4) & 0.053(5) & 0.030(3) & 0.009(4) & 0.004(3) & -0.004(3) \\ \text { C37 } & 0.036(4) & 0.042(4) & 0.030(3) & 0.005(3) & 0.010(3) & 0.003(3) \\ \text { O1' } & 0.055(4) & 0.038(3) & 0.028(3) & 0.015(3) & 0.019(3) & 0.009(2) \\ \text { C34' } & 0.053(5) & 0.039(4) & 0.030(3) & 0.014(4) & 0.006(4) & 0.006(3) \\ \text { C35' } & 0.048(4) & 0.039(4) & 0.026(3) & 0.017(4) & 0.011(4) & 0.001(3) \\ \text { C36' } & 0.046(4) & 0.037(4) & 0.027(3) & 0.005(4) & 0.007(3) & 0.006(3) \\ \text { C37' } & 0.054(4) & 0.054(4) & 0.032(3) & 0.016(4) & 0.010(4) & -0.005(3) \\ & & & & & & \end{array}$

Geometric parameters $\left(\AA,{ }^{\circ}\right)$

\begin{tabular}{llll}
\hline $\mathrm{Ni} 1-\mathrm{C} 1$ & $1.909(3)$ & $\mathrm{C} 22-\mathrm{C} 25$ & $1.500(4)$ \\
$\mathrm{N} i 01-\mathrm{C} 4$ & $1.916(3)$ & $\mathrm{C} 23-\mathrm{H} 23 \mathrm{~A}$ & 0.9800 \\
$\mathrm{~N} i 01-\mathrm{C} 30$ & $2.045(3)$ & $\mathrm{C} 23-\mathrm{H} 23 \mathrm{~B}$ & 0.9800 \\
$\mathrm{~N} i 01-\mathrm{C} 29$ & $2.051(3)$ & $\mathrm{C} 23-\mathrm{H} 23 \mathrm{C}$ & 0.9800 \\
$\mathrm{~N} i 01-\mathrm{C} 26$ & $2.123(3)$ & $\mathrm{C} 24-\mathrm{H} 24 \mathrm{~A}$ & 0.9800 \\
$\mathrm{~N} i 01-\mathrm{C} 33$ & $2.145(3)$ & $\mathrm{C} 24-\mathrm{H} 24 \mathrm{~B}$ & 0.9800 \\
$\mathrm{~N} 1-\mathrm{C} 1$ & $1.377(4)$ & $\mathrm{C} 24-\mathrm{H} 24 \mathrm{C}$ & 0.9800 \\
$\mathrm{~N} 1-\mathrm{C} 2$ & $1.390(4)$ & $\mathrm{C} 25-\mathrm{H} 25 \mathrm{~A}$ & 0.9800 \\
$\mathrm{~N} 1-\mathrm{C} 8$ & $1.437(4)$ & $\mathrm{C} 25-\mathrm{H} 25 \mathrm{~B}$ & 0.9800 \\
$\mathrm{~N} 2-\mathrm{C} 1$ & $1.374(3)$ & $\mathrm{C} 25-\mathrm{H} 25 \mathrm{C}$ & 0.9800 \\
$\mathrm{~N} 2-\mathrm{C} 3$ & $1.384(4)$ & $\mathrm{C} 26-\mathrm{C} 33$ & $1.374(4)$ \\
$\mathrm{N} 2-\mathrm{C} 7$ & $1.451(4)$ & $\mathrm{C} 26-\mathrm{C} 27$ & $1.510(4)$ \\
$\mathrm{N} 3-\mathrm{C} 4$ & $1.379(3)$ & $\mathrm{C} 26-\mathrm{H} 26 \mathrm{~A}$ & $0.951(18)$ \\
$\mathrm{N} 3-\mathrm{C} 5$ & $1.388(3)$ & $\mathrm{C} 27-\mathrm{C} 28$ & $1.536(4)$ \\
$\mathrm{N} 3-\mathrm{C} 7$ & $1.444(3)$ & $\mathrm{C} 27-\mathrm{H} 27 \mathrm{~A}$ & 0.9900 \\
$\mathrm{~N} 4-\mathrm{C} 4$ & $1.374(3)$ & $\mathrm{C} 27-\mathrm{H} 27 \mathrm{~B}$ & 0.9900 \\
$\mathrm{~N} 4-\mathrm{C} 6$ & $1.395(3)$ & $\mathrm{C} 28-\mathrm{C} 29$ & $1.523(4)$ \\
$\mathrm{N} 4-\mathrm{C} 17$ & $1.441(3)$ & $\mathrm{C} 28-\mathrm{H} 28 \mathrm{~A}$ & 0.9900 \\
$\mathrm{C} 2-\mathrm{C} 3$ & $1.344(4)$ & $\mathrm{C} 28-\mathrm{H} 28 \mathrm{~B}$ & 0.9900 \\
$\mathrm{C} 2-\mathrm{H} 2$ & 0.9500 & $\mathrm{C} 29-\mathrm{C} 30$ & $1.411(4)$ \\
$\mathrm{C} 3-\mathrm{H} 3$ & 0.9500 & $\mathrm{C} 29-\mathrm{H} 29 \mathrm{~A}$ & $0.960(18)$
\end{tabular}




\begin{tabular}{|c|c|c|c|}
\hline $\mathrm{C} 5-\mathrm{C} 6$ & $1.341(4)$ & $\mathrm{C} 30-\mathrm{C} 31$ & $1.512(4)$ \\
\hline $\mathrm{C} 5-\mathrm{H} 5$ & 0.9500 & $\mathrm{C} 30-\mathrm{H} 30 \mathrm{~A}$ & $0.956(19)$ \\
\hline $\mathrm{C} 6-\mathrm{H} 6$ & 0.9500 & $\mathrm{C} 31-\mathrm{C} 32$ & $1.539(4)$ \\
\hline C7-H7A & 0.9900 & $\mathrm{C} 31-\mathrm{H} 31 \mathrm{~A}$ & 0.9900 \\
\hline $\mathrm{C} 7-\mathrm{H} 7 \mathrm{~B}$ & 0.9900 & $\mathrm{C} 31-\mathrm{H} 31 \mathrm{~B}$ & 0.9900 \\
\hline $\mathrm{C} 8-\mathrm{C} 13$ & $1.392(4)$ & $\mathrm{C} 32-\mathrm{C} 33$ & $1.516(4)$ \\
\hline $\mathrm{C} 8-\mathrm{C} 9$ & $1.394(4)$ & $\mathrm{C} 32-\mathrm{H} 32 \mathrm{~A}$ & 0.9900 \\
\hline $\mathrm{C} 9-\mathrm{C} 10$ & $1.397(4)$ & $\mathrm{C} 32-\mathrm{H} 32 \mathrm{~B}$ & 0.9900 \\
\hline C9- $\mathrm{C} 14$ & $1.503(4)$ & C $33-\mathrm{H} 33 \mathrm{~A}$ & $0.960(19)$ \\
\hline $\mathrm{C} 10-\mathrm{C} 11$ & $1.379(4)$ & $\mathrm{O} 1-\mathrm{C} 34$ & $1.415(11)$ \\
\hline $\mathrm{C} 10-\mathrm{H} 10$ & 0.9500 & $\mathrm{O} 1-\mathrm{C} 37$ & $1.417(8)$ \\
\hline $\mathrm{C} 11-\mathrm{C} 12$ & $1.392(4)$ & $\mathrm{C} 34-\mathrm{C} 35$ & $1.506(11)$ \\
\hline $\mathrm{C} 11-\mathrm{C} 15$ & $1.510(4)$ & C34-H34A & 0.9900 \\
\hline $\mathrm{C} 12-\mathrm{C} 13$ & $1.387(4)$ & C $34-\mathrm{H} 34 \mathrm{~B}$ & 0.9900 \\
\hline $\mathrm{C} 12-\mathrm{H} 12$ & 0.9500 & $\mathrm{C} 35-\mathrm{C} 36$ & $1.503(12)$ \\
\hline $\mathrm{C} 13-\mathrm{C} 16$ & $1.505(4)$ & $\mathrm{C} 35-\mathrm{H} 35 \mathrm{~A}$ & 0.9900 \\
\hline $\mathrm{C} 14-\mathrm{H} 14 \mathrm{~A}$ & 0.9800 & C $35-\mathrm{H} 35 \mathrm{~B}$ & 0.9900 \\
\hline $\mathrm{C} 14-\mathrm{H} 14 \mathrm{~B}$ & 0.9800 & $\mathrm{C} 36-\mathrm{C} 37$ & $1.512(11)$ \\
\hline $\mathrm{C} 14-\mathrm{H} 14 \mathrm{C}$ & 0.9800 & $\mathrm{C} 36-\mathrm{H} 36 \mathrm{~A}$ & 0.9900 \\
\hline C15-H15A & 0.9800 & $\mathrm{C} 36-\mathrm{H} 36 \mathrm{~B}$ & 0.9900 \\
\hline C15-H15B & 0.9800 & C37-H37A & 0.9900 \\
\hline $\mathrm{C} 15-\mathrm{H} 15 \mathrm{C}$ & 0.9800 & C37-H37B & 0.9900 \\
\hline $\mathrm{C} 16-\mathrm{H} 16 \mathrm{~A}$ & 0.9800 & $\mathrm{O} 1^{\prime}-\mathrm{C} 37^{\prime}$ & $1.344(9)$ \\
\hline C16-H16B & 0.9800 & $\mathrm{O} 1^{\prime}-\mathrm{C} 34^{\prime}$ & $1.420(10)$ \\
\hline $\mathrm{C} 16-\mathrm{H} 16 \mathrm{C}$ & 0.9800 & $\mathrm{C} 34^{\prime}-\mathrm{C} 35^{\prime}$ & $1.524(11)$ \\
\hline $\mathrm{C} 17-\mathrm{C} 18$ & $1.389(4)$ & $\mathrm{C} 34^{\prime}-\mathrm{H} 34 \mathrm{C}$ & 0.9900 \\
\hline $\mathrm{C} 17-\mathrm{C} 22$ & $1.400(4)$ & $\mathrm{C} 34^{\prime}-\mathrm{H} 34 \mathrm{D}$ & 0.9900 \\
\hline $\mathrm{C} 18-\mathrm{C} 19$ & $1.390(4)$ & $\mathrm{C} 35^{\prime}-\mathrm{C} 36^{\prime}$ & $1.526(11)$ \\
\hline $\mathrm{C} 18-\mathrm{C} 23$ & $1.512(4)$ & $\mathrm{C} 35^{\prime}-\mathrm{H} 35 \mathrm{C}$ & 0.9900 \\
\hline $\mathrm{C} 19-\mathrm{C} 20$ & $1.390(4)$ & $\mathrm{C} 35^{\prime}-\mathrm{H} 35 \mathrm{D}$ & 0.9900 \\
\hline C19-H19 & 0.9500 & $\mathrm{C} 36^{\prime}-\mathrm{C} 37^{\prime}$ & $1.514(11)$ \\
\hline $\mathrm{C} 20-\mathrm{C} 21$ & $1.385(4)$ & $\mathrm{C} 36^{\prime}-\mathrm{H} 36 \mathrm{C}$ & 0.9900 \\
\hline $\mathrm{C} 20-\mathrm{C} 24$ & $1.512(4)$ & $\mathrm{C} 36^{\prime}-\mathrm{H} 36 \mathrm{D}$ & 0.9900 \\
\hline $\mathrm{C} 21-\mathrm{C} 22$ & $1.394(4)$ & $\mathrm{C} 37^{\prime}-\mathrm{H} 37 \mathrm{C}$ & 0.9900 \\
\hline $\mathrm{C} 21-\mathrm{H} 21$ & 0.9500 & $\mathrm{C} 37^{\prime}-\mathrm{H} 37 \mathrm{D}$ & 0.9900 \\
\hline $\mathrm{C} 1-\mathrm{Ni} 01-\mathrm{C} 4$ & $91.51(12)$ & $\mathrm{C} 20-\mathrm{C} 24-\mathrm{H} 24 \mathrm{~A}$ & 109.5 \\
\hline $\mathrm{C} 1-\mathrm{Ni} 01-\mathrm{C} 30$ & $107.31(12)$ & $\mathrm{C} 20-\mathrm{C} 24-\mathrm{H} 24 \mathrm{~B}$ & 109.5 \\
\hline $\mathrm{C} 4-\mathrm{Ni} 01-\mathrm{C} 30$ & $142.09(12)$ & $\mathrm{H} 24 \mathrm{~A}-\mathrm{C} 24-\mathrm{H} 24 \mathrm{~B}$ & 109.5 \\
\hline $\mathrm{C} 1-\mathrm{Ni} 01-\mathrm{C} 29$ & $143.24(12)$ & $\mathrm{C} 20-\mathrm{C} 24-\mathrm{H} 24 \mathrm{C}$ & 109.5 \\
\hline $\mathrm{C} 4-\mathrm{Ni01}-\mathrm{C} 29$ & $107.85(12)$ & $\mathrm{H} 24 \mathrm{~A}-\mathrm{C} 24-\mathrm{H} 24 \mathrm{C}$ & 109.5 \\
\hline $\mathrm{C} 30-\mathrm{Ni} 01-\mathrm{C} 29$ & $40.29(11)$ & $\mathrm{H} 24 \mathrm{~B}-\mathrm{C} 24-\mathrm{H} 24 \mathrm{C}$ & 109.5 \\
\hline $\mathrm{C} 1-\mathrm{Ni} 01-\mathrm{C} 26$ & $125.47(12)$ & $\mathrm{C} 22-\mathrm{C} 25-\mathrm{H} 25 \mathrm{~A}$ & 109.5 \\
\hline $\mathrm{C} 4-\mathrm{Ni} 01-\mathrm{C} 26$ & $93.00(12)$ & $\mathrm{C} 22-\mathrm{C} 25-\mathrm{H} 25 \mathrm{~B}$ & 109.5 \\
\hline $\mathrm{C} 30-\mathrm{Ni} 01-\mathrm{C} 26$ & $101.53(12)$ & $\mathrm{H} 25 \mathrm{~A}-\mathrm{C} 25-\mathrm{H} 25 \mathrm{~B}$ & 109.5 \\
\hline $\mathrm{C} 29-\mathrm{Ni} 01-\mathrm{C} 26$ & $85.37(12)$ & $\mathrm{C} 22-\mathrm{C} 25-\mathrm{H} 25 \mathrm{C}$ & 109.5 \\
\hline $\mathrm{C} 1-\mathrm{Ni} 01-\mathrm{C} 33$ & $100.31(12)$ & $\mathrm{H} 25 \mathrm{~A}-\mathrm{C} 25-\mathrm{H} 25 \mathrm{C}$ & 109.5 \\
\hline $\mathrm{C} 4-\mathrm{Ni} 01-\mathrm{C} 33$ & $124.47(12)$ & $\mathrm{H} 25 \mathrm{~B}-\mathrm{C} 25-\mathrm{H} 25 \mathrm{C}$ & 109.5 \\
\hline
\end{tabular}




\begin{tabular}{|c|c|c|c|}
\hline $\mathrm{C} 30-\mathrm{Ni01}-\mathrm{C} 33$ & $84.99(12)$ & $\mathrm{C} 33-\mathrm{C} 26-\mathrm{C} 27$ & $125.9(3)$ \\
\hline $\mathrm{C} 29-\mathrm{Ni01}-\mathrm{C} 33$ & $94.06(12)$ & $\mathrm{C} 33-\mathrm{C} 26-\mathrm{Ni} 01$ & $72.11(18)$ \\
\hline $\mathrm{C} 26-\mathrm{Ni} 01-\mathrm{C} 33$ & $37.55(11)$ & $\mathrm{C} 27-\mathrm{C} 26-\mathrm{Ni0} 1$ & $106.80(19)$ \\
\hline $\mathrm{C} 1-\mathrm{N} 1-\mathrm{C} 2$ & $112.4(2)$ & $\mathrm{C} 33-\mathrm{C} 26-\mathrm{H} 26 \mathrm{~A}$ & $116.6(17)$ \\
\hline $\mathrm{C} 1-\mathrm{N} 1-\mathrm{C} 8$ & $125.1(2)$ & $\mathrm{C} 27-\mathrm{C} 26-\mathrm{H} 26 \mathrm{~A}$ & $115.6(17)$ \\
\hline $\mathrm{C} 2-\mathrm{N} 1-\mathrm{C} 8$ & $122.3(2)$ & $\mathrm{Ni} 01-\mathrm{C} 26-\mathrm{H} 26 \mathrm{~A}$ & $105.1(17)$ \\
\hline $\mathrm{C} 1-\mathrm{N} 2-\mathrm{C} 3$ & $113.4(2)$ & $\mathrm{C} 26-\mathrm{C} 27-\mathrm{C} 28$ & $113.8(3)$ \\
\hline $\mathrm{C} 1-\mathrm{N} 2-\mathrm{C} 7$ & $120.2(2)$ & $\mathrm{C} 26-\mathrm{C} 27-\mathrm{H} 27 \mathrm{~A}$ & 108.8 \\
\hline $\mathrm{C} 3-\mathrm{N} 2-\mathrm{C} 7$ & $126.4(2)$ & $\mathrm{C} 28-\mathrm{C} 27-\mathrm{H} 27 \mathrm{~A}$ & 108.8 \\
\hline $\mathrm{C} 4-\mathrm{N} 3-\mathrm{C} 5$ & $112.8(2)$ & $\mathrm{C} 26-\mathrm{C} 27-\mathrm{H} 27 \mathrm{~B}$ & 108.8 \\
\hline $\mathrm{C} 4-\mathrm{N} 3-\mathrm{C} 7$ & $121.0(2)$ & $\mathrm{C} 28-\mathrm{C} 27-\mathrm{H} 27 \mathrm{~B}$ & 108.8 \\
\hline $\mathrm{C} 5-\mathrm{N} 3-\mathrm{C} 7$ & $126.1(2)$ & $\mathrm{H} 27 \mathrm{~A}-\mathrm{C} 27-\mathrm{H} 27 \mathrm{~B}$ & 107.7 \\
\hline $\mathrm{C} 4-\mathrm{N} 4-\mathrm{C} 6$ & $112.1(2)$ & $\mathrm{C} 29-\mathrm{C} 28-\mathrm{C} 27$ & $112.3(2)$ \\
\hline $\mathrm{C} 4-\mathrm{N} 4-\mathrm{C} 17$ & $126.2(2)$ & $\mathrm{C} 29-\mathrm{C} 28-\mathrm{H} 28 \mathrm{~A}$ & 109.1 \\
\hline $\mathrm{C} 6-\mathrm{N} 4-\mathrm{C} 17$ & $121.7(2)$ & $\mathrm{C} 27-\mathrm{C} 28-\mathrm{H} 28 \mathrm{~A}$ & 109.1 \\
\hline $\mathrm{N} 2-\mathrm{C} 1-\mathrm{N} 1$ & $101.4(2)$ & $\mathrm{C} 29-\mathrm{C} 28-\mathrm{H} 28 \mathrm{~B}$ & 109.1 \\
\hline $\mathrm{N} 2-\mathrm{C} 1-\mathrm{Ni01}$ & $119.8(2)$ & $\mathrm{C} 27-\mathrm{C} 28-\mathrm{H} 28 \mathrm{~B}$ & 109.1 \\
\hline $\mathrm{N} 1-\mathrm{C} 1-\mathrm{Ni01}$ & $138.6(2)$ & $\mathrm{H} 28 \mathrm{~A}-\mathrm{C} 28-\mathrm{H} 28 \mathrm{~B}$ & 107.9 \\
\hline $\mathrm{C} 3-\mathrm{C} 2-\mathrm{N} 1$ & $106.9(3)$ & $\mathrm{C} 30-\mathrm{C} 29-\mathrm{C} 28$ & $122.4(3)$ \\
\hline $\mathrm{C} 3-\mathrm{C} 2-\mathrm{H} 2$ & 126.5 & $\mathrm{C} 30-\mathrm{C} 29-\mathrm{Ni01}$ & $69.64(16)$ \\
\hline $\mathrm{N} 1-\mathrm{C} 2-\mathrm{H} 2$ & 126.5 & $\mathrm{C} 28-\mathrm{C} 29-\mathrm{Ni0} 1$ & $111.99(19)$ \\
\hline $\mathrm{C} 2-\mathrm{C} 3-\mathrm{N} 2$ & $105.9(3)$ & $\mathrm{C} 30-\mathrm{C} 29-\mathrm{H} 29 \mathrm{~A}$ & $119.2(17)$ \\
\hline $\mathrm{C} 2-\mathrm{C} 3-\mathrm{H} 3$ & 127.0 & $\mathrm{C} 28-\mathrm{C} 29-\mathrm{H} 29 \mathrm{~A}$ & $113.7(17)$ \\
\hline $\mathrm{N} 2-\mathrm{C} 3-\mathrm{H} 3$ & 127.0 & $\mathrm{Ni} 01-\mathrm{C} 29-\mathrm{H} 29 \mathrm{~A}$ & $109.8(17)$ \\
\hline $\mathrm{N} 4-\mathrm{C} 4-\mathrm{N} 3$ & $101.8(2)$ & $\mathrm{C} 29-\mathrm{C} 30-\mathrm{C} 31$ & $123.1(3)$ \\
\hline $\mathrm{N} 4-\mathrm{C} 4-\mathrm{Ni01}$ & $139.2(2)$ & $\mathrm{C} 29-\mathrm{C} 30-\mathrm{Ni01}$ & $70.07(16)$ \\
\hline $\mathrm{N} 3-\mathrm{C} 4-\mathrm{Ni01}$ & $119.02(19)$ & $\mathrm{C} 31-\mathrm{C} 30-\mathrm{Ni01}$ & $111.30(19)$ \\
\hline $\mathrm{C} 6-\mathrm{C} 5-\mathrm{N} 3$ & $106.2(2)$ & $\mathrm{C} 29-\mathrm{C} 30-\mathrm{H} 30 \mathrm{~A}$ & $116.0(19)$ \\
\hline $\mathrm{C} 6-\mathrm{C} 5-\mathrm{H} 5$ & 126.9 & $\mathrm{C} 31-\mathrm{C} 30-\mathrm{H} 30 \mathrm{~A}$ & $116.3(19)$ \\
\hline $\mathrm{N} 3-\mathrm{C} 5-\mathrm{H} 5$ & 126.9 & $\mathrm{Ni} 01-\mathrm{C} 30-\mathrm{H} 30 \mathrm{~A}$ & $108.9(19)$ \\
\hline $\mathrm{C} 5-\mathrm{C} 6-\mathrm{N} 4$ & $107.1(3)$ & $\mathrm{C} 30-\mathrm{C} 31-\mathrm{C} 32$ & $113.9(2)$ \\
\hline $\mathrm{C} 5-\mathrm{C} 6-\mathrm{H} 6$ & 126.5 & $\mathrm{C} 30-\mathrm{C} 31-\mathrm{H} 31 \mathrm{~A}$ & 108.8 \\
\hline $\mathrm{N} 4-\mathrm{C} 6-\mathrm{H} 6$ & 126.5 & $\mathrm{C} 32-\mathrm{C} 31-\mathrm{H} 31 \mathrm{~A}$ & 108.8 \\
\hline $\mathrm{N} 3-\mathrm{C} 7-\mathrm{N} 2$ & $110.2(2)$ & $\mathrm{C} 30-\mathrm{C} 31-\mathrm{H} 31 \mathrm{~B}$ & 108.8 \\
\hline $\mathrm{N} 3-\mathrm{C} 7-\mathrm{H} 7 \mathrm{~A}$ & 109.6 & $\mathrm{C} 32-\mathrm{C} 31-\mathrm{H} 31 \mathrm{~B}$ & 108.8 \\
\hline $\mathrm{N} 2-\mathrm{C} 7-\mathrm{H} 7 \mathrm{~A}$ & 109.6 & $\mathrm{H} 31 \mathrm{~A}-\mathrm{C} 31-\mathrm{H} 31 \mathrm{~B}$ & 107.7 \\
\hline $\mathrm{N} 3-\mathrm{C} 7-\mathrm{H} 7 \mathrm{~B}$ & 109.6 & $\mathrm{C} 33-\mathrm{C} 32-\mathrm{C} 31$ & $114.1(2)$ \\
\hline $\mathrm{N} 2-\mathrm{C} 7-\mathrm{H} 7 \mathrm{~B}$ & 109.6 & $\mathrm{C} 33-\mathrm{C} 32-\mathrm{H} 32 \mathrm{~A}$ & 108.7 \\
\hline $\mathrm{H} 7 \mathrm{~A}-\mathrm{C} 7-\mathrm{H} 7 \mathrm{~B}$ & 108.1 & $\mathrm{C} 31-\mathrm{C} 32-\mathrm{H} 32 \mathrm{~A}$ & 108.7 \\
\hline $\mathrm{C} 13-\mathrm{C} 8-\mathrm{C} 9$ & $121.6(3)$ & $\mathrm{C} 33-\mathrm{C} 32-\mathrm{H} 32 \mathrm{~B}$ & 108.7 \\
\hline $\mathrm{C} 13-\mathrm{C} 8-\mathrm{N} 1$ & $117.8(3)$ & $\mathrm{C} 31-\mathrm{C} 32-\mathrm{H} 32 \mathrm{~B}$ & 108.7 \\
\hline $\mathrm{C} 9-\mathrm{C} 8-\mathrm{N} 1$ & $120.5(3)$ & $\mathrm{H} 32 \mathrm{~A}-\mathrm{C} 32-\mathrm{H} 32 \mathrm{~B}$ & 107.6 \\
\hline $\mathrm{C} 8-\mathrm{C} 9-\mathrm{C} 10$ & $117.5(3)$ & $\mathrm{C} 26-\mathrm{C} 33-\mathrm{C} 32$ & $123.8(3)$ \\
\hline $\mathrm{C} 8-\mathrm{C} 9-\mathrm{C} 14$ & $121.9(3)$ & $\mathrm{C} 26-\mathrm{C} 33-\mathrm{Ni01}$ & $70.34(17)$ \\
\hline $\mathrm{C} 10-\mathrm{C} 9-\mathrm{C} 14$ & $120.6(3)$ & $\mathrm{C} 32-\mathrm{C} 33-\mathrm{Ni01}$ & $109.86(19)$ \\
\hline $\mathrm{C} 11-\mathrm{C} 10-\mathrm{C} 9$ & $122.4(3)$ & $\mathrm{C} 26-\mathrm{C} 33-\mathrm{H} 33 \mathrm{~A}$ & $118.2(17)$ \\
\hline $\mathrm{C} 11-\mathrm{C} 10-\mathrm{H} 10$ & 118.8 & $\mathrm{C} 32-\mathrm{C} 33-\mathrm{H} 33 \mathrm{~A}$ & $115.3(17)$ \\
\hline $\mathrm{C} 9-\mathrm{C} 10-\mathrm{H} 10$ & 118.8 & $\mathrm{Ni} 01-\mathrm{C} 33-\mathrm{H} 33 \mathrm{~A}$ & $106.4(17)$ \\
\hline
\end{tabular}




\begin{tabular}{|c|c|c|c|}
\hline $\mathrm{C} 10-\mathrm{C} 11-\mathrm{C} 12$ & $118.3(3)$ & $\mathrm{C} 34-\mathrm{O} 1-\mathrm{C} 37$ & $110.5(7)$ \\
\hline $\mathrm{C} 10-\mathrm{C} 11-\mathrm{C} 15$ & $122.1(3)$ & $\mathrm{O} 1-\mathrm{C} 34-\mathrm{C} 35$ & $107.6(9)$ \\
\hline $\mathrm{C} 12-\mathrm{C} 11-\mathrm{C} 15$ & $119.6(3)$ & $\mathrm{O} 1-\mathrm{C} 34-\mathrm{H} 34 \mathrm{~A}$ & 110.2 \\
\hline $\mathrm{C} 13-\mathrm{C} 12-\mathrm{C} 11$ & $121.5(3)$ & $\mathrm{C} 35-\mathrm{C} 34-\mathrm{H} 34 \mathrm{~A}$ & 110.2 \\
\hline $\mathrm{C} 13-\mathrm{C} 12-\mathrm{H} 12$ & 119.3 & $\mathrm{O} 1-\mathrm{C} 34-\mathrm{H} 34 \mathrm{~B}$ & 110.2 \\
\hline $\mathrm{C} 11-\mathrm{C} 12-\mathrm{H} 12$ & 119.3 & $\mathrm{C} 35-\mathrm{C} 34-\mathrm{H} 34 \mathrm{~B}$ & 110.2 \\
\hline $\mathrm{C} 12-\mathrm{C} 13-\mathrm{C} 8$ & $118.6(3)$ & $\mathrm{H} 34 \mathrm{~A}-\mathrm{C} 34-\mathrm{H} 34 \mathrm{~B}$ & 108.5 \\
\hline $\mathrm{C} 12-\mathrm{C} 13-\mathrm{C} 16$ & $120.4(3)$ & $\mathrm{C} 36-\mathrm{C} 35-\mathrm{C} 34$ & $103.4(8)$ \\
\hline $\mathrm{C} 8-\mathrm{C} 13-\mathrm{C} 16$ & $120.9(3)$ & $\mathrm{C} 36-\mathrm{C} 35-\mathrm{H} 35 \mathrm{~A}$ & 111.1 \\
\hline $\mathrm{C} 9-\mathrm{C} 14-\mathrm{H} 14 \mathrm{~A}$ & 109.5 & $\mathrm{C} 34-\mathrm{C} 35-\mathrm{H} 35 \mathrm{~A}$ & 111.1 \\
\hline C9-C14-H14B & 109.5 & $\mathrm{C} 36-\mathrm{C} 35-\mathrm{H} 35 \mathrm{~B}$ & 111.1 \\
\hline $\mathrm{H} 14 \mathrm{~A}-\mathrm{C} 14-\mathrm{H} 14 \mathrm{~B}$ & 109.5 & $\mathrm{C} 34-\mathrm{C} 35-\mathrm{H} 35 \mathrm{~B}$ & 111.1 \\
\hline C9- $\mathrm{C} 14-\mathrm{H} 14 \mathrm{C}$ & 109.5 & $\mathrm{H} 35 \mathrm{~A}-\mathrm{C} 35-\mathrm{H} 35 \mathrm{~B}$ & 109.1 \\
\hline $\mathrm{H} 14 \mathrm{~A}-\mathrm{C} 14-\mathrm{H} 14 \mathrm{C}$ & 109.5 & $\mathrm{C} 35-\mathrm{C} 36-\mathrm{C} 37$ & $103.9(9)$ \\
\hline $\mathrm{H} 14 \mathrm{~B}-\mathrm{C} 14-\mathrm{H} 14 \mathrm{C}$ & 109.5 & $\mathrm{C} 35-\mathrm{C} 36-\mathrm{H} 36 \mathrm{~A}$ & 111.0 \\
\hline $\mathrm{C} 11-\mathrm{C} 15-\mathrm{H} 15 \mathrm{~A}$ & 109.5 & $\mathrm{C} 37-\mathrm{C} 36-\mathrm{H} 36 \mathrm{~A}$ & 111.0 \\
\hline $\mathrm{C} 11-\mathrm{C} 15-\mathrm{H} 15 \mathrm{~B}$ & 109.5 & $\mathrm{C} 35-\mathrm{C} 36-\mathrm{H} 36 \mathrm{~B}$ & 111.0 \\
\hline $\mathrm{H} 15 \mathrm{~A}-\mathrm{C} 15-\mathrm{H} 15 \mathrm{~B}$ & 109.5 & $\mathrm{C} 37-\mathrm{C} 36-\mathrm{H} 36 \mathrm{~B}$ & 111.0 \\
\hline $\mathrm{C} 11-\mathrm{C} 15-\mathrm{H} 15 \mathrm{C}$ & 109.5 & $\mathrm{H} 36 \mathrm{~A}-\mathrm{C} 36-\mathrm{H} 36 \mathrm{~B}$ & 109.0 \\
\hline $\mathrm{H} 15 \mathrm{~A}-\mathrm{C} 15-\mathrm{H} 15 \mathrm{C}$ & 109.5 & $\mathrm{O} 1-\mathrm{C} 37-\mathrm{C} 36$ & $106.3(7)$ \\
\hline $\mathrm{H} 15 \mathrm{~B}-\mathrm{C} 15-\mathrm{H} 15 \mathrm{C}$ & 109.5 & $\mathrm{O} 1-\mathrm{C} 37-\mathrm{H} 37 \mathrm{~A}$ & 110.5 \\
\hline $\mathrm{C} 13-\mathrm{C} 16-\mathrm{H} 16 \mathrm{~A}$ & 109.5 & $\mathrm{C} 36-\mathrm{C} 37-\mathrm{H} 37 \mathrm{~A}$ & 110.5 \\
\hline $\mathrm{C} 13-\mathrm{C} 16-\mathrm{H} 16 \mathrm{~B}$ & 109.5 & $\mathrm{O} 1-\mathrm{C} 37-\mathrm{H} 37 \mathrm{~B}$ & 110.5 \\
\hline $\mathrm{H} 16 \mathrm{~A}-\mathrm{C} 16-\mathrm{H} 16 \mathrm{~B}$ & 109.5 & $\mathrm{C} 36-\mathrm{C} 37-\mathrm{H} 37 \mathrm{~B}$ & 110.5 \\
\hline $\mathrm{C} 13-\mathrm{C} 16-\mathrm{H} 16 \mathrm{C}$ & 109.5 & $\mathrm{H} 37 \mathrm{~A}-\mathrm{C} 37-\mathrm{H} 37 \mathrm{~B}$ & 108.7 \\
\hline $\mathrm{H} 16 \mathrm{~A}-\mathrm{C} 16-\mathrm{H} 16 \mathrm{C}$ & 109.5 & $\mathrm{C} 37^{\prime}-\mathrm{O} 1^{\prime}-\mathrm{C} 34^{\prime}$ & $110.2(7)$ \\
\hline $\mathrm{H} 16 \mathrm{~B}-\mathrm{C} 16-\mathrm{H} 16 \mathrm{C}$ & 109.5 & $\mathrm{O} 1^{\prime}-\mathrm{C} 34^{\prime}-\mathrm{C} 35^{\prime}$ & $105.3(8)$ \\
\hline $\mathrm{C} 18-\mathrm{C} 17-\mathrm{C} 22$ & $121.9(3)$ & $\mathrm{O} 1^{\prime}-\mathrm{C} 34^{\prime}-\mathrm{H} 34 \mathrm{C}$ & 110.7 \\
\hline $\mathrm{C} 18-\mathrm{C} 17-\mathrm{N} 4$ & $119.4(2)$ & $\mathrm{C} 35^{\prime}-\mathrm{C} 34^{\prime}-\mathrm{H} 34 \mathrm{C}$ & 110.7 \\
\hline $\mathrm{C} 22-\mathrm{C} 17-\mathrm{N} 4$ & $118.6(3)$ & $\mathrm{O} 1^{\prime}-\mathrm{C} 34^{\prime}-\mathrm{H} 34 \mathrm{D}$ & 110.7 \\
\hline $\mathrm{C} 17-\mathrm{C} 18-\mathrm{C} 19$ & $118.2(3)$ & $\mathrm{C} 35^{\prime}-\mathrm{C} 34^{\prime}-\mathrm{H} 34 \mathrm{D}$ & 110.7 \\
\hline $\mathrm{C} 17-\mathrm{C} 18-\mathrm{C} 23$ & $122.2(3)$ & $\mathrm{H} 34 \mathrm{C}-\mathrm{C} 34^{\prime}-\mathrm{H} 34 \mathrm{D}$ & 108.8 \\
\hline $\mathrm{C} 19-\mathrm{C} 18-\mathrm{C} 23$ & $119.6(3)$ & $\mathrm{C} 34^{\prime}-\mathrm{C} 35^{\prime}-\mathrm{C} 36^{\prime}$ & $102.8(9)$ \\
\hline $\mathrm{C} 18-\mathrm{C} 19-\mathrm{C} 20$ & $121.8(3)$ & $\mathrm{C} 34^{\prime}-\mathrm{C} 35^{\prime}-\mathrm{H} 35 \mathrm{C}$ & 111.2 \\
\hline $\mathrm{C} 18-\mathrm{C} 19-\mathrm{H} 19$ & 119.1 & $\mathrm{C} 36^{\prime}-\mathrm{C} 35^{\prime}-\mathrm{H} 35 \mathrm{C}$ & 111.2 \\
\hline $\mathrm{C} 20-\mathrm{C} 19-\mathrm{H} 19$ & 119.1 & $\mathrm{C} 34^{\prime}-\mathrm{C} 35^{\prime}-\mathrm{H} 35 \mathrm{D}$ & 111.2 \\
\hline $\mathrm{C} 21-\mathrm{C} 20-\mathrm{C} 19$ & $118.4(3)$ & $\mathrm{C} 36^{\prime}-\mathrm{C} 35^{\prime}-\mathrm{H} 35 \mathrm{D}$ & 111.2 \\
\hline $\mathrm{C} 21-\mathrm{C} 20-\mathrm{C} 24$ & $120.7(3)$ & $\mathrm{H} 35 \mathrm{C}-\mathrm{C} 35^{\prime}-\mathrm{H} 35 \mathrm{D}$ & 109.1 \\
\hline $\mathrm{C} 19-\mathrm{C} 20-\mathrm{C} 24$ & $120.8(3)$ & $\mathrm{C} 37^{\prime}-\mathrm{C} 36^{\prime}-\mathrm{C} 35^{\prime}$ & $100.3(8)$ \\
\hline $\mathrm{C} 20-\mathrm{C} 21-\mathrm{C} 22$ & $122.0(3)$ & $\mathrm{C} 37^{\prime}-\mathrm{C} 36^{\prime}-\mathrm{H} 36 \mathrm{C}$ & 111.7 \\
\hline $\mathrm{C} 20-\mathrm{C} 21-\mathrm{H} 21$ & 119.0 & $\mathrm{C} 35^{\prime}-\mathrm{C} 36^{\prime}-\mathrm{H} 36 \mathrm{C}$ & 111.7 \\
\hline $\mathrm{C} 22-\mathrm{C} 21-\mathrm{H} 21$ & 119.0 & $\mathrm{C} 37^{\prime}-\mathrm{C} 36^{\prime}-\mathrm{H} 36 \mathrm{D}$ & 111.7 \\
\hline $\mathrm{C} 21-\mathrm{C} 22-\mathrm{C} 17$ & $117.6(3)$ & $\mathrm{C} 35^{\prime}-\mathrm{C} 36^{\prime}-\mathrm{H} 36 \mathrm{D}$ & 111.7 \\
\hline $\mathrm{C} 21-\mathrm{C} 22-\mathrm{C} 25$ & $120.8(3)$ & $\mathrm{H} 36 \mathrm{C}-\mathrm{C} 36^{\prime}-\mathrm{H} 36 \mathrm{D}$ & 109.5 \\
\hline $\mathrm{C} 17-\mathrm{C} 22-\mathrm{C} 25$ & $121.5(3)$ & $\mathrm{O} 1^{\prime}-\mathrm{C} 37^{\prime}-\mathrm{C} 36^{\prime}$ & $111.1(7)$ \\
\hline $\mathrm{C} 18-\mathrm{C} 23-\mathrm{H} 23 \mathrm{~A}$ & 109.5 & $\mathrm{O} 1^{\prime}-\mathrm{C} 37^{\prime}-\mathrm{H} 37 \mathrm{C}$ & 109.4 \\
\hline $\mathrm{C} 18-\mathrm{C} 23-\mathrm{H} 23 \mathrm{~B}$ & 109.5 & $\mathrm{C} 36^{\prime}-\mathrm{C} 37^{\prime}-\mathrm{H} 37 \mathrm{C}$ & 109.4 \\
\hline $\mathrm{H} 23 \mathrm{~A}-\mathrm{C} 23-\mathrm{H} 23 \mathrm{~B}$ & 109.5 & $\mathrm{O} 1^{\prime}-\mathrm{C} 37^{\prime}-\mathrm{H} 37 \mathrm{D}$ & 109.4 \\
\hline
\end{tabular}




\begin{tabular}{|c|c|}
\hline $\mathrm{C} 18-\mathrm{C} 23-\mathrm{H} 23 \mathrm{C}$ & 109.5 \\
\hline $\mathrm{H} 23 \mathrm{~A}-\mathrm{C} 23-\mathrm{H} 23 \mathrm{C}$ & 109.5 \\
\hline $\mathrm{H} 23 \mathrm{~B}-\mathrm{C} 23-\mathrm{H} 23 \mathrm{C}$ & 109.5 \\
\hline $\mathrm{C} 3-\mathrm{N} 2-\mathrm{C} 1-\mathrm{N} 1$ & $-0.4(3)$ \\
\hline $\mathrm{C} 7-\mathrm{N} 2-\mathrm{C} 1-\mathrm{N} 1$ & $-179.3(2)$ \\
\hline $\mathrm{C} 3-\mathrm{N} 2-\mathrm{C} 1-\mathrm{Ni} 01$ & $-175.9(2)$ \\
\hline $\mathrm{C} 7-\mathrm{N} 2-\mathrm{C} 1-\mathrm{Ni} 01$ & $5.2(3)$ \\
\hline $\mathrm{C} 2-\mathrm{N} 1-\mathrm{C} 1-\mathrm{N} 2$ & $0.8(3)$ \\
\hline $\mathrm{C} 8-\mathrm{N} 1-\mathrm{C} 1-\mathrm{N} 2$ & $175.4(3)$ \\
\hline $\mathrm{C} 2-\mathrm{N} 1-\mathrm{C} 1-\mathrm{Ni} 01$ & $174.8(3)$ \\
\hline $\mathrm{C} 8-\mathrm{N} 1-\mathrm{C} 1-\mathrm{Ni} 01$ & $-10.5(5)$ \\
\hline $\mathrm{C} 1-\mathrm{N} 1-\mathrm{C} 2-\mathrm{C} 3$ & $-0.8(3)$ \\
\hline $\mathrm{C} 8-\mathrm{N} 1-\mathrm{C} 2-\mathrm{C} 3$ & $-175.7(3)$ \\
\hline $\mathrm{N} 1-\mathrm{C} 2-\mathrm{C} 3-\mathrm{N} 2$ & $0.5(3)$ \\
\hline $\mathrm{C} 1-\mathrm{N} 2-\mathrm{C} 3-\mathrm{C} 2$ & $-0.1(3)$ \\
\hline $\mathrm{C} 7-\mathrm{N} 2-\mathrm{C} 3-\mathrm{C} 2$ & $178.8(3)$ \\
\hline $\mathrm{C} 6-\mathrm{N} 4-\mathrm{C} 4-\mathrm{N} 3$ & $-0.8(3)$ \\
\hline $\mathrm{C} 17-\mathrm{N} 4-\mathrm{C} 4-\mathrm{N} 3$ & $176.5(2)$ \\
\hline $\mathrm{C} 6-\mathrm{N} 4-\mathrm{C} 4-\mathrm{Ni} 01$ & $178.4(3)$ \\
\hline $\mathrm{C} 17-\mathrm{N} 4-\mathrm{C} 4-\mathrm{Ni} 01$ & $-4.3(5)$ \\
\hline $\mathrm{C} 5-\mathrm{N} 3-\mathrm{C} 4-\mathrm{N} 4$ & $0.6(3)$ \\
\hline $\mathrm{C} 7-\mathrm{N} 3-\mathrm{C} 4-\mathrm{N} 4$ & $178.6(2)$ \\
\hline $\mathrm{C} 5-\mathrm{N} 3-\mathrm{C} 4-\mathrm{Ni} 01$ & $-178.8(2)$ \\
\hline $\mathrm{C} 7-\mathrm{N} 3-\mathrm{C} 4-\mathrm{Ni} 01$ & $-0.8(4)$ \\
\hline $\mathrm{C} 4-\mathrm{N} 3-\mathrm{C} 5-\mathrm{C} 6$ & $-0.2(3)$ \\
\hline $\mathrm{C} 7-\mathrm{N} 3-\mathrm{C} 5-\mathrm{C} 6$ & $-178.1(3)$ \\
\hline $\mathrm{N} 3-\mathrm{C} 5-\mathrm{C} 6-\mathrm{N} 4$ & $-0.3(3)$ \\
\hline $\mathrm{C} 4-\mathrm{N} 4-\mathrm{C} 6-\mathrm{C} 5$ & $0.7(3)$ \\
\hline $\mathrm{C} 17-\mathrm{N} 4-\mathrm{C} 6-\mathrm{C} 5$ & $-176.7(3)$ \\
\hline $\mathrm{C} 4-\mathrm{N} 3-\mathrm{C} 7-\mathrm{N} 2$ & $53.4(3)$ \\
\hline $\mathrm{C} 5-\mathrm{N} 3-\mathrm{C} 7-\mathrm{N} 2$ & $-128.9(3)$ \\
\hline $\mathrm{C} 1-\mathrm{N} 2-\mathrm{C} 7-\mathrm{N} 3$ & $-56.0(3)$ \\
\hline $\mathrm{C} 3-\mathrm{N} 2-\mathrm{C} 7-\mathrm{N} 3$ & $125.3(3)$ \\
\hline $\mathrm{C} 1-\mathrm{N} 1-\mathrm{C} 8-\mathrm{C} 13$ & $-91.4(3)$ \\
\hline $\mathrm{C} 2-\mathrm{N} 1-\mathrm{C} 8-\mathrm{C} 13$ & $82.8(4)$ \\
\hline $\mathrm{C} 1-\mathrm{N} 1-\mathrm{C} 8-\mathrm{C} 9$ & $90.7(4)$ \\
\hline $\mathrm{C} 2-\mathrm{N} 1-\mathrm{C} 8-\mathrm{C} 9$ & $-95.1(3)$ \\
\hline $\mathrm{C} 13-\mathrm{C} 8-\mathrm{C} 9-\mathrm{C} 10$ & $0.7(4)$ \\
\hline $\mathrm{N} 1-\mathrm{C} 8-\mathrm{C} 9-\mathrm{C} 10$ & $178.6(3)$ \\
\hline $\mathrm{C} 13-\mathrm{C} 8-\mathrm{C} 9-\mathrm{C} 14$ & $-178.9(3)$ \\
\hline $\mathrm{N} 1-\mathrm{C} 8-\mathrm{C} 9-\mathrm{C} 14$ & $-1.1(4)$ \\
\hline $\mathrm{C} 8-\mathrm{C} 9-\mathrm{C} 10-\mathrm{C} 11$ & $-1.8(4)$ \\
\hline $\mathrm{C} 14-\mathrm{C} 9-\mathrm{C} 10-\mathrm{C} 11$ & $177.9(3)$ \\
\hline $\mathrm{C} 9-\mathrm{C} 10-\mathrm{C} 11-\mathrm{C} 12$ & $1.4(4)$ \\
\hline $\mathrm{C} 9-\mathrm{C} 10-\mathrm{C} 11-\mathrm{C} 15$ & $-178.1(3)$ \\
\hline $\mathrm{C} 10-\mathrm{C} 11-\mathrm{C} 12-\mathrm{C} 13$ & $0.1(4)$ \\
\hline $\mathrm{C} 15-\mathrm{C} 11-\mathrm{C} 12-\mathrm{C} 13$ & $179.6(3)$ \\
\hline
\end{tabular}

C $36^{\prime}-\mathrm{C} 37^{\prime}-\mathrm{H} 37 \mathrm{D}$

H37C-C $37^{\prime}-\mathrm{H} 37 \mathrm{D}$

C9-C8-C13-C16

$\mathrm{N} 1-\mathrm{C} 8-\mathrm{C} 13-\mathrm{C} 16$

$\mathrm{C} 4-\mathrm{N} 4-\mathrm{C} 17-\mathrm{C} 18$

C6-N4-C17-C18

$\mathrm{C} 4-\mathrm{N} 4-\mathrm{C} 17-\mathrm{C} 22$

C6-N4-C17-C22

$\mathrm{C} 22-\mathrm{C} 17-\mathrm{C} 18-\mathrm{C} 19$

N4-C17-C18-C19

$\mathrm{C} 22-\mathrm{C} 17-\mathrm{C} 18-\mathrm{C} 23$

$\mathrm{N} 4-\mathrm{C} 17-\mathrm{C} 18-\mathrm{C} 23$

$\mathrm{C} 17-\mathrm{C} 18-\mathrm{C} 19-\mathrm{C} 20$

$\mathrm{C} 23-\mathrm{C} 18-\mathrm{C} 19-\mathrm{C} 20$

$\mathrm{C} 18-\mathrm{C} 19-\mathrm{C} 20-\mathrm{C} 21$

$\mathrm{C} 18-\mathrm{C} 19-\mathrm{C} 20-\mathrm{C} 24$

$\mathrm{C} 19-\mathrm{C} 20-\mathrm{C} 21-\mathrm{C} 22$

$\mathrm{C} 24-\mathrm{C} 20-\mathrm{C} 21-\mathrm{C} 22$

$\mathrm{C} 20-\mathrm{C} 21-\mathrm{C} 22-\mathrm{C} 17$

$\mathrm{C} 20-\mathrm{C} 21-\mathrm{C} 22-\mathrm{C} 25$

$\mathrm{C} 18-\mathrm{C} 17-\mathrm{C} 22-\mathrm{C} 21$

$\mathrm{N} 4-\mathrm{C} 17-\mathrm{C} 22-\mathrm{C} 21$

$\mathrm{C} 18-\mathrm{C} 17-\mathrm{C} 22-\mathrm{C} 25$

$\mathrm{N} 4-\mathrm{C} 17-\mathrm{C} 22-\mathrm{C} 25$

$\mathrm{C} 33-\mathrm{C} 26-\mathrm{C} 27-\mathrm{C} 28$

$\mathrm{Ni01}-\mathrm{C} 26-\mathrm{C} 27-\mathrm{C} 28$

$\mathrm{C} 26-\mathrm{C} 27-\mathrm{C} 28-\mathrm{C} 29$

$\mathrm{C} 27-\mathrm{C} 28-\mathrm{C} 29-\mathrm{C} 30$

$\mathrm{C} 27-\mathrm{C} 28-\mathrm{C} 29-\mathrm{Ni} 01$

$\mathrm{C} 28-\mathrm{C} 29-\mathrm{C} 30-\mathrm{C} 31$

$\mathrm{Ni} 01-\mathrm{C} 29-\mathrm{C} 30-\mathrm{C} 31$

$\mathrm{C} 28-\mathrm{C} 29-\mathrm{C} 30-\mathrm{Ni} 01$

$\mathrm{C} 29-\mathrm{C} 30-\mathrm{C} 31-\mathrm{C} 32$

$\mathrm{Ni} 01-\mathrm{C} 30-\mathrm{C} 31-\mathrm{C} 32$

$\mathrm{C} 30-\mathrm{C} 31-\mathrm{C} 32-\mathrm{C} 33$

$\mathrm{C} 27-\mathrm{C} 26-\mathrm{C} 33-\mathrm{C} 32$

$\mathrm{Ni} 01-\mathrm{C} 26-\mathrm{C} 33-\mathrm{C} 32$

$\mathrm{C} 27-\mathrm{C} 26-\mathrm{C} 33-\mathrm{Ni} 01$

$\mathrm{C} 31-\mathrm{C} 32-\mathrm{C} 33-\mathrm{C} 26$

$\mathrm{C} 31-\mathrm{C} 32-\mathrm{C} 33-\mathrm{Ni} 01$

$\mathrm{C} 37-\mathrm{O} 1-\mathrm{C} 34-\mathrm{C} 35$

$\mathrm{O} 1-\mathrm{C} 34-\mathrm{C} 35-\mathrm{C} 36$

$\mathrm{C} 34-\mathrm{C} 35-\mathrm{C} 36-\mathrm{C} 37$

$\mathrm{C} 34-\mathrm{O} 1-\mathrm{C} 37-\mathrm{C} 36$

$\mathrm{C} 35-\mathrm{C} 36-\mathrm{C} 37-\mathrm{O} 1$

$\mathrm{C} 37^{\prime}-\mathrm{O}^{\prime}-\mathrm{C} 34^{\prime}-\mathrm{C} 35^{\prime}$
109.4

108.0

$-177.7(3)$

$4.4(4)$

$115.5(3)$

$-67.4(4)$

-67.7 (4)

$109.3(3)$

$0.2(4)$

176.8 (2)

$-177.6(3)$

$-0.9(4)$

$-0.4(4)$

$177.4(3)$

$0.8(4)$

$-178.3(3)$

$-1.1(4)$

$178.0(3)$

0.9 (4)

-179.5 (3)

$-0.4(4)$

-177.1 (2)

-180.0 (3)

$3.4(4)$

$-46.3(4)$

$33.5(3)$

$-32.1(4)$

$92.8(3)$

$13.6(3)$

-0.7 (4)

$103.0(3)$

$-103.7(3)$

$-52.8(4)$

26.7 (3)

$-24.3(4)$

$-3.1(5)$

$-101.2(3)$

$98.1(3)$

89.1 (4)

$10.0(3)$

6.5 (17)

$-22.2(19)$

28.4 (18)

12.0 (16)

$-25.4(16)$

-17.7 (17) 


\begin{tabular}{llll}
$\mathrm{C} 11-\mathrm{C} 12-\mathrm{C} 13-\mathrm{C} 8$ & $-1.1(4)$ & $\mathrm{O} 1^{\prime}-\mathrm{C} 34^{\prime}-\mathrm{C} 35^{\prime}-\mathrm{C} 36^{\prime}$ & $30.1(18)$ \\
$\mathrm{C} 11-\mathrm{C} 12-\mathrm{C} 13-\mathrm{C} 16$ & $177.3(3)$ & $\mathrm{C} 34^{\prime}-\mathrm{C} 35^{\prime}-\mathrm{C} 36^{\prime}-\mathrm{C} 37^{\prime}$ & $-29.8(17)$ \\
$\mathrm{C} 9-\mathrm{C} 8-\mathrm{C} 13-\mathrm{C} 12$ & $0.7(4)$ & $\mathrm{C} 34^{\prime}-\mathrm{O} 1^{\prime}-\mathrm{C} 37^{\prime}-\mathrm{C} 36^{\prime}$ & $-2.5(16)$ \\
$\mathrm{N} 1-\mathrm{C} 8-\mathrm{C} 13-\mathrm{C} 12$ & $-177.2(3)$ & $\mathrm{C} 35^{\prime}-\mathrm{C} 36^{\prime}-\mathrm{C} 37^{\prime}-\mathrm{O} 1^{\prime}$ & $21.2(17)$ \\
\hline
\end{tabular}

